\title{
16.
}

\section{De orbitis cometarum ex observationibus determinandis commentatio.}

\author{
(Auct. Dr. A. T. Bergius, ad Acad. Upsaliens. Docens Astronomiae.)
}

Ad quaestiones nostrae aetati gravissimas Astronomiae ea sine dubio est referenda, quae in natura et motu cometarum indagandis et in orbitis illorum ex observationibus determinandis versalur, quod problema summus jam Newton in principiis Philosophiae naturalis difficillimum nuncupavit. Post Newton viri celeberrimi Euler, Laplace, Lagrange, Olbers, Legendre varias hujus problematis solvendi vias inierunt. Methodus nunc temporis maxime usitata, quae etiam simplicitate sua brevitateque nescio an omnibus aliis palmam praeripiat, est ea Cel. Olbers ${ }^{1}$ ), quae, formulis a Cel. Gau $\left(s^{2}\right.$ ) ad simplicissimam formam reductis, viam nobis aperuit brevissimam, qua ad notitiam approximativam orbilae cometae ex tribus observationibus geocentricis pervenire possumus. His methodis elementa parabolica cometae observati invenimus. Quum vero maxime probabile est, cometas, qui nostra aelate observentur, in orbitis ellipticis moveri ${ }^{3}$ ), et quum ex cometis super cenlum et quinquaginta jam observatis ${ }^{4}$ ) elementa elliptica quattuor tantummodo a Cel. Halley, Olbers, Encke et Biela diversis temporibus computata sunt, summo jure dicere possumus, nos a vera cognitione orbitarum plerorumque cometarum valde esse remotos, ne de natura et indole horum corporum coelestium loquamur; quamvis post summum Newton, qui sagacilate sua animi superstitiosum illum horum corporum terrorem removit, etiam haec pars Astronomiae nostris certe temporibus maximis progressibus se gaudere possit.

Post tantos viros, qui in his disquisitionibus summo studio sunt versali, multis haud procul a temeritate remotum videri potest, nos, specimen

1) Abhandlung über die leichteste und bequenste Methode die Bahn eines Cometen zu berechnen. Weimar 1797.

2) v. Zach. Monatliche Correspondenz zur Beförderung der Erd - und Himmelshunde XXVIII. Band pag. 501.

3) Laplace, Traite de Mécanique céleste. Tome I ${ }^{\text {pr }}$ p. 232.

+) Catalogum optinum orbitarum omnium cometarum adhuc computatarum viri Cell. Schumacher. et Olbers tradiderunt, in particula prima tet tertia in ,Schumacher's Astronomischen Al,handlungen." Orbitae cometarum post 1825 computatae in variis numeris ,der Astronomischen Nachrichten" a Schumacher sunt contentate.

Crelle's Journal f. d. M. Bd. XXV, Heft 3. 
190 16. Bergius, de orbitis cometarum ex observationibus determin. commentatio.

Astronomicum edituros, istam materiam potissimum elegisse, nisi in eo excusationem quamdam haberemus, quod novus ab Laugier Parisiis his ipsis temporibus cometa inventus, summum in nobis hujus rei pertraclandae erexit siudium. Indulgentiae igilur Astronomorum has studiorum nostrorum recommendamus primilias.

\$. 1.

Ad problema de orbitis comelarum determinandis plane solvendum in genere tres observaliones requiruntur, et sane omni rigore solvi posset, nisi aequationes, ad quas hac via ducimur, gradus tam elevali essent et lam complicalae, ut ad methodos approximalionis nos confugere necesse sil.

Si corpus coeleste nostri systematis solaris consideremus, cujus distantia a sole $\boldsymbol{r}$ est, cujusque positionem in spatio determinemus coordinatis reclangulis $x, y, z$, quorum originem in centro solis ponamus, si distantiam mediam solis a tellure unitatem distantiae ponamus, si porro $\mu$ summam massarum solis: alteriusque corporis coelestis exprimat, et vim altractivam solis ut unicam in hoc agentem consideremus; hae relationes ${ }^{1}$ ) salis sunt cognilae

$$
\frac{d^{2} x}{d t^{2}}+\frac{x \mu}{r^{3}}=0, \quad \frac{d^{2} y}{d t^{2}}+\frac{y \mu}{r^{3}}=0, \quad \frac{d^{2} z}{d t^{2}}+\frac{z \mu}{r^{3}}=0 . \quad \text {. (1), }
$$

E quibus sine ullo negotio tres integrales primos deducimus sequentes

$$
\frac{\| d z-z d y}{d t}=a, \quad \frac{z d x-x d z}{d t}=b, \quad \frac{x d y-y d x}{d t}=c .
$$

ubi $a, b$ et $c$ conslantes arbitrarias integratione inductas exprimunt. Si tres aequationes (2) respective cum $x, y$ et $z$ multiplicemus, addilione oblinebimus

$$
a x+b y+c z=0 .
$$

Quocirca concludimus, orbitam, in qua corpus coeleste movelur, in plano esse sitam, quae per centrum solis transit.

\$. 2.

Si aequationes (1) respective cum $2 d x, 2 d y$ et $2 d \approx$ multiplicemus additione et integratione, designante $h$ constantem arbitrariam, oblinebimus

$$
\frac{d x^{2}+d y^{2}+d z^{2}}{d t^{2}}=\frac{2 \mu}{r}-h
$$

1) Laplace, Méc. cell. 'Toune I ${ }^{\mathrm{Pr}}$ pag. $15 \%$ 
cujus valoris substitutione in aequatione

$$
\frac{r^{2}\left(d x^{2}+d y^{2}+d z^{2}\right)}{d t^{2}}-\frac{r^{2} d r^{2}}{d t^{2}}=a^{2}+b^{2}+c^{2}
$$

quae ex aequationibus (2) stalim sequitur, si relationem observemus $r^{2}=$ $x^{2}+y^{2}+z^{2}$; prodibil hic ipsius $d t$ valor

$$
d t=\frac{r d r}{\sqrt{\left(2 \mu r-h r^{2}-k^{2}\right)}} \cdot . \quad . \quad . \quad .
$$

ubi hrevilatis gratia $a^{2}+b_{2}+c^{2}=k^{2}$ posuimus.

Designet $\boldsymbol{d} v$ angulum infinite parvum inter duos radios veclores consecutivos, erit

$$
d x^{2}+d y^{2}+d z^{2}=d r^{2}+r^{2} d v^{2}
$$

quibus in (3) substitutis, relatio haecce inter areas et tempora interque celeritatem angularem $\frac{d v}{d t}$ et radium vectorem satis cognita prodibit

$$
d v=\frac{i d t}{r^{2}}
$$

Si pro $d t$ valorem suum ex (4) substituamus, prodibit

$$
d v=\frac{l d r}{r V\left(2 \mu r-h r^{2}-k^{2}\right)} .
$$

ex qua aequationem polarem orbitae integratione obtinebimus. Ex illa aequatione maximus et minimus ipsius $\boldsymbol{r}$ valor determinatur hac relatione $2 \mu r-h r^{2}-k^{2}=0$. Si igitur maximum ipsius $r$ valorem per $a(1+e)$, minimum vero per $a(1-e)$ exprimamus, erit summa horum valorum $\frac{2 \mu}{h}$ et produclum $\frac{k^{2}}{h}$, quo pro constantibus $k$ et $h$ sequentes obtinebimus valores

$$
k=r \mu v\left(a\left(1-e^{2}\right)\right), \quad l=\frac{\mu}{a}
$$

quibus substitutis aequatio differentialis hanc induit formam

$$
d v=\frac{V\left(a\left(1-e^{2}\right)\right) d r}{r \sqrt{ }\left(2 r-\frac{1}{a} r^{2}-a\left(1-e^{2}\right)\right)}
$$

Illa iniegrata sub positione, $v$ evanescere, quum $r=a(1-e)$, efficit

$$
r=\frac{a\left(1-e^{2}\right)}{1+e \cos v}
$$

quae est aequatio sectionum conicarum, origine coordinatarum in altero foco posita, in qua $e$ excentricitatem et $v$ angulum, quem radius vector cnm axi majori $\boldsymbol{a}$ facit, a Perihelio computatum, exprimunt. 
192 16. Bergius, de orbitis cometarum ex obscrvationibus determin. commentatio.

\$. 3.

Si orbita esset valde excentrica, ut cometarum semper est, formulae molus elliplici in series convergentes facillime evolvuntur. Quum hoc casu $e$ ab unitate parum differat, aequatio $(b)$; distanlia minima $a(1-e)=q$ posita, in hanc abit

$$
\begin{gathered}
r=\frac{q}{\cos ^{2} \frac{1}{2} v\left(1+\frac{1-e}{1+c} \operatorname{tang}^{2} \frac{1}{2} v\right)}, \text { et evolutione facta } \\
r=\frac{q}{\cos ^{2} \frac{1}{2} v}\left\{1-\frac{1-c}{1+e} \operatorname{tang}^{2} \frac{1}{2} v+\left(\frac{1-c}{1+e}\right)^{2} \operatorname{tang}^{4} \frac{1}{2} v-\left(\frac{1-c}{1+e}\right)^{3} \operatorname{lang}^{6} \frac{1}{2} v+\text { elc. }\right\}
\end{gathered}
$$

Hoc valore pro $r$ in (5) substituto prodibit

$$
\begin{aligned}
& d t=\frac{2 q^{2}}{\sqrt{\left(\mu a\left(1-c^{2}\right)\right)}}\left(1+\operatorname{tang}^{2} \frac{1}{2} v\right)\left\{1-\frac{1-e}{1+e} \operatorname{tang}^{2} \frac{1}{2} v+\left(\frac{1-e}{1+e}\right)^{2} \operatorname{tang}^{+} \frac{1}{2} v\right. \\
& \left.-\left(\frac{1-e}{1+c}\right)^{3} \operatorname{tang}^{6} \frac{1}{2} v+e l c \cdot\right\}^{2} \cdot \frac{\frac{1}{2} d v}{\cos ^{2} \frac{1}{2} v}
\end{aligned}
$$

et evolulione et integratione sub positione $t$ evanescere, quum $v=0$,

$$
\begin{aligned}
t=\frac{2 y^{2}}{v^{-}\left(\mu a\left(1-c^{2}\right)\right)}\left\{\operatorname{tang} \frac{1}{2} v\right. & -\frac{2\left(\frac{1-e}{1+c}\right)-1}{3} \operatorname{tang}^{3} \frac{1}{2} v \\
+ & \left.\frac{\frac{1-e}{1+c}\left(3\left(\frac{1-e}{1+c}\right)-2\right)}{5} \operatorname{tang}^{5} \frac{1}{2} v \ldots \text { etc. }\right\}
\end{aligned}
$$

Si orbila in parabolam abiret, erit $e=1$ et $\checkmark\left(\mu_{a}\left(1-e^{2}\right)\right)=\checkmark(2 \mu q)$, quo igitur casu formulae pro $r$ et $t$ hanc induunt formam simplicissimam

$$
\left.\begin{array}{l}
r=\frac{q}{\cos ^{2} \frac{1}{2} v} \\
t=\frac{q^{\frac{3}{2}} V 2}{V \mu}\left(\operatorname{tang} \frac{1}{2} v+\frac{1}{3} \operatorname{tang}^{3} \frac{1}{2} v\right)
\end{array}\right\}
$$

Designantibus $\boldsymbol{r}^{\prime}$ et $\boldsymbol{v}^{\prime}$ radium vectorem et anomaliam veram, quae lempori $t^{\prime}$ conveniunt, erit $t^{\prime}-t$ tempus, quo cometa arcum parabolicum, inter duos radios vectores contentum, describit. Posita igilur massa solis $=1$, quantitate aulem materiae cometae respectu solis neglecta ${ }^{1}$ ) erit $\mu_{0}=1$, et ideo

1) Jus hujus suppositionis nobis concedunt infinite parvae massae omnium cometarum olsservatorum. Vide inter alios Littrow ,über den gefürchteten Cometen des Jahres 1832 und über die Cometen überhaupt. Wien 1832. pag. 38." 
16. Bergius, de orbitis cometarum ex observationibus determin. commentatio. 193

$$
\left.\begin{array}{cc}
t^{\prime}-t=r\left(2 q^{3}\right)\left(\operatorname{tang} \frac{1}{2} v^{\prime}-\operatorname{tang} \frac{1}{2} v+\frac{1}{3}\left(\operatorname{tang}^{3} \frac{1}{2} v^{\prime}-\operatorname{tang}^{3} \frac{1}{2} v\right)\right) \\
\boldsymbol{r}=q\left(1+\operatorname{lang}^{2} \frac{1}{2} v\right), & r^{\prime}=q\left(1+\operatorname{tang}^{2} \frac{1}{2} v^{\prime}\right)
\end{array}\right\}
$$

Si $c$ chordam exprimit arcus hujus, quem radii vectores $\boldsymbol{r}$ et $\boldsymbol{r}^{\prime}$ subsecant, erit

$$
\begin{aligned}
c^{2} & =r^{2}+r^{2}-2 r r^{\prime} \cos \left(v^{\prime}-v\right) \\
& =r^{2}+r^{\prime 2}-2 r r^{\prime} \frac{1-\operatorname{tang}^{2} \frac{1}{2}\left(v^{\prime}-v\right)}{1+\operatorname{tang}^{2} \frac{1}{2}\left(v^{\prime}-v\right)} \\
& =\left(r+r^{\prime}\right)^{2}-\frac{4 r r^{\prime}}{1+\operatorname{tang}^{2} \frac{1}{2}\left(v^{\prime}-v\right)},
\end{aligned}
$$

quapropter

$$
\frac{r r^{\prime}}{1+\operatorname{tang}^{2} \frac{1}{2}} \frac{\left.1 v^{\prime}-v\right)}{4}=\frac{1}{4}\left(\boldsymbol{r}+\boldsymbol{r}^{\prime}+c\right)\left(\boldsymbol{r}+\boldsymbol{r}^{\prime}-c\right) \text {. }
$$

Quum vero sit

$$
\operatorname{tang}_{\frac{2}{2}} v^{\prime}=\frac{\operatorname{tang} \frac{1}{2}\left(v^{\prime}-v\right)+\operatorname{tang} \frac{1}{2} v}{1-\operatorname{tang} \frac{1}{2}\left(v^{\prime}-v\right) \operatorname{tang} \frac{1}{2} v}, \quad r=q\left(1+\operatorname{tang}^{2} \frac{1}{2} v\right),
$$

erit

$$
\begin{gathered}
r^{\prime}=q\left(1+\operatorname{tang}^{2} \frac{1}{2} v^{\prime}\right)=\frac{r\left(1+\operatorname{tang}^{2} \frac{1}{2}\left(v^{\prime}-v\right)\right)}{\left(1-\operatorname{tang} \frac{1}{2}\left(v^{\prime}-v\right) \operatorname{tang} \frac{1}{2} v\right)^{2}}, \\
\frac{1}{4}\left(r+r^{\prime}+c\right)\left(r+r^{\prime}-c\right)=\frac{q^{2}\left(1+\operatorname{tang}^{2} \frac{1}{2} v\right)^{2}}{\left(1-\operatorname{tang} \frac{1}{2}\left(v^{\prime}-v\right) \operatorname{tang} \frac{1}{2} v\right)^{2}} .
\end{gathered}
$$

Si brevitatis gralia ponamus $\frac{1}{2}\left(r+r^{\prime}+c\right)=m, \frac{1}{2}\left(r+r^{\prime}-c\right)=n$, erit

$$
\begin{aligned}
& m+n=\frac{2 q\left(1+\operatorname{tang}^{2} \frac{1}{2} v\right)}{1-\operatorname{tang} \frac{1}{2}\left(v^{\prime}-v\right) \operatorname{tang} \frac{1}{2} v}+\frac{q \operatorname{tang}^{2} \frac{1}{2}\left(v^{\prime}-v\right)\left(1+\operatorname{tang}^{2} \frac{1}{2} v\right)^{2}}{\left(1-\operatorname{tang} \frac{1}{2}\left(v^{\prime}-v\right) \operatorname{tang} \frac{1}{2} v\right)^{2}} \\
& = \pm 2 \sqrt{ }(m n)+\operatorname{tang}^{2} \frac{1}{2}\left(v^{\prime}-v\right) \frac{m n}{q}
\end{aligned}
$$

ideoque

$$
\operatorname{tang} \frac{1}{2}\left(v^{\prime}-v\right)=r q \frac{r_{m} \mp r n}{V(m n)} .
$$

Expressio (11) pro $t^{\prime}-t$ etiam sequenti modo scribi potest

$t^{\prime}-t=\sqrt{ }\left(2 q^{3}\right) \frac{\operatorname{tang} \frac{1}{2}\left(v^{\prime}-v\right)\left(1+\operatorname{tang}^{2} \frac{1}{2} v\right)^{2}}{\left(1-\operatorname{tang} \frac{1}{2}\left(v^{\prime}-v\right) \operatorname{tang} \frac{1}{2} v\right)^{2}}\left\{1+\frac{1}{3} \frac{\operatorname{tang}^{2} \frac{1}{2}\left(v^{\prime}-v\right)\left(1+\operatorname{tang}^{2} \frac{1}{2} v\right)}{1-\operatorname{tang} \frac{1}{2}\left(v^{\prime}-v\right) \operatorname{tang} \frac{1}{2} v}\right\}$ quae valoribus jam inventos introductis in hanc abit formam

$$
t^{\prime}-t=\frac{V_{2}}{3}\left(m^{\frac{3}{2}} \mp n^{\frac{3}{2}}\right)=\frac{1}{6}\left(r+r^{\prime}+c\right)^{\frac{3}{2}} \mp \frac{1}{6}\left(r+r^{\prime}-c\right)^{\frac{3}{2}} .
$$

Si tempus $t^{\prime}-t$ in diebus expressum desideramus, hoc per constantem in theoria motus $k=0,0172021$, cujus logarithmus est $\log k=8,2355814$, multiplicari necesse est. ${ }^{1}$ )

1) Gau/s, Theoria motus corporum coelestium pag. 2. 
194 16. Bergivs, de orbitis cometarum ex observationibus determin. commentatio.

Expressio illa elegans, quae tempus per duos radios veclores et chordam dat, a Cel. Euler inventa ${ }^{1}$ ), primum a Cel. Lambert ${ }^{2}$ ) fuil applicata. Signum superius vel inferius valet, prout $v^{\prime}-v$ infra vel supra $180^{\circ}$ est.

S. 4 .

Si $x y z, x^{\prime} y^{\prime} z^{\prime}, x^{\prime \prime} y^{\prime \prime} z^{\prime \prime}$ coordinatas trium punctorum in orbita corporis coelestis, quae in plano per centrum solis transeunte est sita, designent. tres sequentes aequationes ( $\$$. 1.) habebimus,

$$
\begin{aligned}
& a x+b y+c z=0 \\
& a x^{\prime}+b y^{\prime}+c z^{\prime}=0 \\
& a x^{\prime \prime}+b y^{\prime \prime}+c z^{\prime \prime}=0
\end{aligned}
$$

ex quibus eliminatione duarum quantitatum $\frac{b}{a}$ et $\frac{c}{a}$ sequentem aequationem oblinemus conditionalem

$$
\left(y^{\prime \prime} z^{\prime}-y^{\prime} z^{\prime \prime}\right) x+\left(y z^{\prime \prime}-y^{\prime \prime} z\right) x^{\prime}+\left(y^{\prime} z-y z^{\prime}\right) x^{\prime \prime}=0 .
$$

Quum vero $y^{\prime \prime} \mathfrak{z}^{\prime}-y^{\prime} z^{\prime \prime}$ projectionem in planum $y z$ areac duplicis trianguli exprimit, qui per centrum solis et puncta $x^{\prime} y^{\prime} z^{\prime}, x^{\prime \prime} y^{\prime \prime} z^{\prime \prime}$ determinatur, si aream hujus trianguli duplicem per $\left[\boldsymbol{r}^{\prime} \boldsymbol{r}^{\prime \prime}\right]$, sicuti triangulorum, quos radii vectores $\boldsymbol{r}$ et $\boldsymbol{r}^{\prime \prime}$, et $\boldsymbol{r}$ et $\boldsymbol{r}^{\prime}$ formant, per $\left[\boldsymbol{r} \boldsymbol{r}^{\prime \prime}\right]$ et $\left[\boldsymbol{r} \boldsymbol{r}^{\prime}\right]$ designemus, aequatio illa condilionalis formas tres induit hasce, prout $\boldsymbol{b}$ et $\boldsymbol{c}, \boldsymbol{a}$ et $c$, vel $\boldsymbol{a}$ et $\boldsymbol{b}$ eliminantur

$$
\left.\begin{array}{l}
{\left[\boldsymbol{r}^{\prime} \boldsymbol{r}^{\prime \prime}\right] x-\left[\boldsymbol{r} \boldsymbol{r}^{\prime \prime}\right] \boldsymbol{x}^{\prime}+\left[\boldsymbol{r} \boldsymbol{r}^{\prime}\right] \boldsymbol{x}^{\prime \prime}=0} \\
{\left[\boldsymbol{r}^{\prime} \boldsymbol{r}^{\prime \prime}\right] y-\left[\boldsymbol{r} \boldsymbol{r}^{\prime \prime}\right] y^{\prime}+\left[\boldsymbol{r} \boldsymbol{r}^{\prime}\right] y^{\prime \prime}=0} \\
{\left[\boldsymbol{r}^{\prime} \boldsymbol{r}^{\prime \prime}\right] z-\left[\boldsymbol{r} \boldsymbol{r}^{\prime \prime}\right] \boldsymbol{z}^{\prime}+\left[\boldsymbol{r} \boldsymbol{r}^{\prime}\right] \boldsymbol{z}^{\prime \prime}=0}
\end{array}\right\}
$$

quac aequationes sub tribus formis diversis eandem exprimunt conditionem.

Posito :

$\rho, \rho^{\prime}, \rho^{\prime \prime}$ tres distantiae curtatae cometae a tellure;

$\alpha, \alpha^{\prime}, \alpha^{\prime \prime}$ - longitudines geocentricae observatae;

$\delta, \delta^{\prime}, \delta^{\prime \prime}$ - latitudines geocentricae;

$\odot, \odot \odot^{\prime}, \odot^{\prime \prime}$ - longitudines solis;

$\boldsymbol{R}, \boldsymbol{R}^{\prime}, \boldsymbol{R}^{\prime \prime}$ - distantiae telluris a sole;

$t, t^{\prime}, t^{\prime \prime}$ - tempora observationum,

1) Lconh. Euler, Theorie der Planeten und Cometen, von Johann Freiherrn von Pacicassi übersetzt und mit einem Anhange und Tafeln vermehrt. Wien 1781. pag. 148.

2) Lambert, Insigniores orbitae cometarum proprietates Augustae Vindelicorum 1761 pag. 40. Cfr. Laplace, Traité de mécanique céleste. Toune $I^{\text {er }}$ pag. 196, et Gau/s, Theoria inotus corporum coelestium pag. 123. 
16. Bergius, de orbitis cometarum ex observationibus determin. commentatio. 195

erit

$$
\begin{aligned}
& x=\rho \cos \alpha-\boldsymbol{R} \cos \odot, \quad \boldsymbol{y}=\rho \sin \alpha-\boldsymbol{R} \sin \odot, \quad \boldsymbol{z}=\rho \operatorname{tang} \delta, \\
& x^{\prime}=\rho^{\prime} \cos \alpha^{\prime}-\boldsymbol{R}^{\prime} \cos \odot, \quad y^{\prime}=\rho^{\prime} \sin \alpha^{\prime}-\boldsymbol{R}^{\prime} \sin \odot{ }^{\prime}, \quad \boldsymbol{z}^{\prime}=\rho^{\prime} \text { lang } \delta^{\prime}, \\
& x^{\prime \prime}=\rho^{\prime \prime} \cos \alpha^{\prime \prime}-\boldsymbol{R}^{\prime \prime} \cos \odot ", \quad \boldsymbol{y}^{\prime \prime}=\rho^{\prime \prime} \sin \alpha^{\prime \prime}-\boldsymbol{R}^{\prime \prime} \sin \odot{ }^{\prime \prime}, \quad \boldsymbol{z}^{\prime \prime}=\rho^{\prime \prime} \operatorname{tang} \delta^{\prime \prime} \text {. }
\end{aligned}
$$

His valoribus in aequationibus (12) substitulis obtinemus

$\left[\boldsymbol{r}^{\prime} \boldsymbol{r}^{\prime \prime}\right](\rho \cos \alpha-\boldsymbol{R} \cos \odot)-\left[\boldsymbol{r} \boldsymbol{r}^{\prime \prime}\right]\left(\rho^{\prime} \cos \alpha^{\prime}-\boldsymbol{R}^{\prime} \cos \odot{ }^{\prime}\right)+\left[\boldsymbol{r} \boldsymbol{r}^{\prime}\right]\left(\zeta^{\prime \prime} \cos \alpha^{\prime \prime}-\boldsymbol{R}^{\prime \prime} \cos \odot^{\prime \prime}\right)=0$, $\left.\left.\mid \boldsymbol{r}^{\prime} \boldsymbol{r}^{\prime \prime}\right]^{\prime} \rho \sin \alpha-\boldsymbol{R} \sin \odot\right)-\left|\boldsymbol{r} \boldsymbol{r}^{\prime \prime}\right|\left(\rho^{\prime} \sin \alpha^{\prime}-\boldsymbol{R}^{\prime} \sin \odot\right)+\left[\boldsymbol{r} \boldsymbol{r}^{\prime}\right]\left(\rho^{\prime \prime} \sin \alpha^{\prime \prime}-\boldsymbol{R}^{\prime \prime} \sin \odot^{\prime \prime}\right)=\mathbf{0}$, $\left|\boldsymbol{r}^{\prime} \boldsymbol{r}^{\prime \prime}\right| \rho \operatorname{lang} \delta-\left[\boldsymbol{r} \boldsymbol{r}^{\prime \prime}\right] \rho^{\prime} \operatorname{lang} \delta^{\prime}+\left[\boldsymbol{r} \boldsymbol{r}^{\prime}\right] \rho^{\prime \prime} \operatorname{tang} \delta^{\prime \prime}=0$.

Ex his tribus aequationibus tres quantitatum quinque incognitarum $\varrho, \rho^{\prime}, \varrho^{\prime \prime}$, $\left[r^{\prime \prime} r^{\prime \prime}\right], \frac{\left[r^{\prime \prime}\right]}{\left[r^{\prime}\right]}$ eliminatione removere possumus. Ad quem finem si secundam cum $\cos \odot^{\prime}$ multiplicemus et ex producto primam per $\sin \odot^{\prime}$ mulliplicalam detrahamus, ad hanc aequationem ducimur

$$
\begin{aligned}
{\left[\boldsymbol{r}^{\prime} \boldsymbol{r}^{\prime \prime}\right]\left(\rho \sin \left(\alpha-\odot^{\prime}\right)+\boldsymbol{R}\right.} & \left.\sin \left(\odot^{\prime}-\odot\right)\right)-\left[\boldsymbol{r} \boldsymbol{r}^{\prime \prime}\right] \rho^{\prime} \sin \left(\alpha^{\prime}-\odot^{\prime}\right) \\
& +\left[\boldsymbol{r} \boldsymbol{r}^{\prime}\right]\left(\rho^{\prime \prime} \sin \left(\alpha^{\prime \prime}-\odot^{\prime}\right)-\boldsymbol{R}^{\prime \prime} \sin \left(\odot^{\prime \prime}-\odot^{\prime}\right)\right)=0 .
\end{aligned}
$$

Valore ipsius $\left.\mid r \boldsymbol{r}^{\prime \prime}\right] \rho^{\prime}$ ex aequatione tertia deducto in hoc substituto, proveniel hace ipsius $\rho^{\prime \prime}$ expressio

$$
\begin{aligned}
\rho^{\prime \prime} & =\frac{\left[r^{\prime \prime} r^{\prime \prime}\right]}{\left[r^{\prime}\right]} \cdot \frac{\operatorname{tang} \delta^{\prime} \sin \left(\alpha-\odot^{\prime}\right)-\operatorname{tang} \delta \sin \left(\alpha^{\prime}-\odot^{\prime}\right)}{\operatorname{tang} \delta^{\prime \prime} \sin \left(\alpha^{\prime}-\odot^{\prime}\right)-\operatorname{tang} \delta^{\prime} \sin \left(\alpha^{\prime \prime}-\odot^{\prime}\right)} \rho \\
& +\frac{\operatorname{tang} \delta^{\prime}}{\left[r^{\prime}\right]} \cdot \frac{\left[r^{\prime} r^{\prime \prime}\right] R \sin \left(\odot^{\prime}-\odot\right)-\left[r^{\prime}\right] R^{\prime \prime} \sin \left(\odot^{\prime \prime}-\odot^{\prime}\right)}{\operatorname{tang} \delta^{\prime \prime} \sin \left(\alpha^{\prime}-\odot^{\prime}\right)-\operatorname{tang} \delta \sin \left(\alpha^{\prime \prime}-\odot^{\prime}\right)} .
\end{aligned}
$$

Ullimum hujus expressionis membrum simpliciorem induit formam, si eliam pro tellure eundem* significalionis modum introducamus, ut habeamus $\left|\boldsymbol{R}^{\prime} \boldsymbol{R}^{\prime \prime}\right|=\boldsymbol{R}^{\prime} \boldsymbol{R}^{\prime \prime} \sin \left(\odot^{\prime \prime}-\odot^{\prime}\right),\left[\boldsymbol{R} \boldsymbol{R}^{\prime \prime}\right]=\boldsymbol{R} \boldsymbol{R}^{\prime \prime} \sin \left(\odot_{\prime \prime}-\odot\right),\left[\boldsymbol{R} \boldsymbol{R}^{\prime}\right]=\boldsymbol{R} \boldsymbol{R}^{\prime} \sin \left(\odot^{\prime}-\odot\right)$.

Sil porro brevilalis gratia

prodibit

$$
\left.\begin{array}{l}
M^{\prime}=\frac{\operatorname{tang} \delta^{\prime} \sin \left(\alpha-\odot^{\prime}\right)-\operatorname{tang} \delta \sin \left(\alpha^{\prime}-\odot^{\prime}\right)}{\operatorname{tang} \delta^{\prime \prime} \sin \left(\alpha^{\prime}-\odot^{\prime}\right)-\operatorname{tang} \delta^{\prime} \sin \left(\alpha^{\prime \prime}-\odot^{\prime}\right)} \\
M^{\prime \prime}=\frac{\operatorname{tang} \delta^{\prime} \sin \left(\odot^{\prime}-\odot\right)}{\operatorname{tang} \delta^{\prime \prime} \sin \left(\alpha^{\prime}-\odot^{\prime}\right)-\operatorname{tang} \delta^{\prime} \sin \left(\alpha^{\prime \prime}-\odot^{\prime}\right)}
\end{array}\right\}
$$

$$
\zeta^{\prime \prime}=\frac{\left[r^{\prime \prime} r^{\prime \prime}\right]}{\left[r^{\prime \prime}\right]} \cdot \boldsymbol{M}^{\prime} \zeta+\left(\frac{\left[r^{\prime \prime} r^{\prime \prime}\right]}{\left[r r^{\prime \prime}\right]}-\frac{\left[\boldsymbol{R}^{\prime} \boldsymbol{R}^{\prime \prime}\right]}{\left[\boldsymbol{R} \boldsymbol{R}^{\prime}\right]}\right) \boldsymbol{M}^{\prime \prime} \boldsymbol{R} . \quad \cdot(\boldsymbol{f}) .
$$

Quamcunque seclionem conicam cometa describat, eam in parte orbitae, in qua nobis est visibilis, parabolae sese applicantem considerare possumus. Quum vero, ut vidimus, areae, quas radius vector diversis describit temporibus, his ipsis temporibus sint proportionales, et areae triangulorum duplices $\left[\boldsymbol{r}^{\prime} \boldsymbol{r}^{\prime \prime}\right]$, $\left[\boldsymbol{r} \boldsymbol{r}^{\prime}\right]$ parvis temporum intervallis a sectoribus parabolicis correspondentibus 
196 16. Bergius, de orbitis comctarum cx observationibus delermin. commentatio.

parum differant, eoque minus duorum triangulorum ratio a ratione sectorum parabolicorum sint diversi, quumque, quod ad orbitam telluris adtinel, parva excentricitate orbitae ejus considerata, id fere omni rigore pro intervallis temporum aequalibus dicere possimus; sine magno errore statuere possumus:

$$
\frac{t^{\prime \prime}-t^{\prime}}{t^{\prime}-t}=\frac{\left[r^{\prime} r^{\prime \prime}\right]}{\left[r r^{\prime}\right]}=\frac{\left[\boldsymbol{R}^{\prime} R^{\prime \prime}\right]}{\left[\boldsymbol{R R} R^{\prime}\right]}
$$

el. si $\boldsymbol{M}=\frac{t^{\prime \prime}-t^{\prime}}{t^{\prime}-t} \boldsymbol{M}^{\prime}$, expressionem $(f)$ ad formam hanc simplicissimam reducimus

$$
\rho^{\prime \prime}=\frac{t^{\prime \prime}-t^{\prime}}{t^{\prime}-t} M^{\prime} \rho=M \rho
$$

quae rationem inter $\rho$ et $\rho^{\prime \prime}$ in quantitatibus cognitis suppeditat.

\section{§. 5 .}

Ut radios vectores $\boldsymbol{r}, \boldsymbol{r}^{\prime \prime}$ et chordam correspondentem $\boldsymbol{c}$ per eandem quantitatem $\rho$ exprimamus, has notamus relationes $r^{2}=x^{2}+y^{2}+z^{2}, r^{\prime \prime 2}=x^{\prime \prime 2}+y^{\prime \prime 2}+z^{\prime \prime 2}, c^{2}=\left(x^{\prime \prime}-x\right)^{2}+\left(y^{\prime \prime}-y\right)^{2}+\left(z^{\prime \prime}-z\right)^{2}$. Si pro $x, y, z, x^{\prime \prime}, y^{\prime \prime}, z^{\prime \prime}$ valores suos introducamus et observemus, esse $\varrho^{\prime \prime}=M_{\zeta}$, expressiones sequentes sponte prodeunt

$$
\begin{aligned}
r & =\sqrt{ }\left(\frac{\varrho^{2}}{\cos ^{2} \delta}-2 \boldsymbol{R}_{\rho} \cos (\alpha-\odot)+\boldsymbol{R}^{2}\right) \\
\boldsymbol{r}^{\prime \prime} & =\sqrt{ }\left(\frac{\boldsymbol{M}^{2} \varrho^{2}}{\cos ^{2} \delta^{\prime \prime}}-2 \boldsymbol{M} \boldsymbol{R}^{\prime \prime} \rho \cos \left(\alpha^{\prime \prime}-\odot^{\prime \prime}\right)+\boldsymbol{R}^{\prime \prime 2}\right),
\end{aligned}
$$

quae, positis

$$
\begin{aligned}
\cos \delta \cos (\alpha-\odot) & =\cos \psi, & \cos \delta^{\prime \prime} \cos \left(\alpha^{\prime \prime}-\odot^{\prime \prime}\right) & =\cos \psi^{\prime \prime} \\
\boldsymbol{R} \sin \psi & =\boldsymbol{B}, & \boldsymbol{R}^{\prime \prime} \sin \psi^{\prime \prime} & =\boldsymbol{B}^{\prime \prime} .
\end{aligned}
$$

in has abeunt calculo numerico commodiores

$$
\left.\begin{array}{l}
\boldsymbol{r}=\sqrt[V]{ }\left\{\left(\frac{\varrho}{\cos \delta}-\boldsymbol{R} \cos \psi\right)^{2}+\boldsymbol{B}^{2}\right\} \\
\boldsymbol{r}^{\prime \prime}=\sqrt[V]{ }\left\{\left(\frac{\boldsymbol{M} \varrho}{\cos \delta^{\prime \prime}}-\boldsymbol{R}^{\prime \prime} \cos \psi^{\prime \prime}\right)^{2}+\boldsymbol{B}^{\prime \prime 2}\right\}
\end{array}\right\}
$$

Ut pro chorda $c$ commodam obtineamus expressionem, postquam valores ipsorum $x, y, z, x^{\prime \prime}, y^{\prime \prime}, z^{\prime \prime}$ fuerunt substitutae, quinque quantitates auxiliares $g, \boldsymbol{G}, \boldsymbol{h}, \boldsymbol{H}, \zeta$ computamus ex aequationibus hisce suppositis

$$
\left.\begin{array}{l}
\boldsymbol{R}^{\prime \prime} \cos \odot{ }^{\prime \prime}-\boldsymbol{R} \cos \odot=\boldsymbol{g} \cos \boldsymbol{G}, \text { vel } \boldsymbol{R}^{\prime \prime} \cos \left(\odot^{\prime \prime}-\odot\right)-\boldsymbol{R}=\boldsymbol{g} \cos (\boldsymbol{G}-\odot) \boldsymbol{r} \\
\boldsymbol{R}^{\prime \prime} \sin \odot \odot^{\prime \prime}-\boldsymbol{R} \sin \odot=\boldsymbol{g} \sin \boldsymbol{G}, \text { vel } \boldsymbol{R}^{\prime \prime} \sin \left(\odot^{\prime \prime}-\odot\right) \quad \boldsymbol{g} \sin (\boldsymbol{G}-\odot)
\end{array}\right\}
$$

$\boldsymbol{M} \cos \alpha^{\prime \prime}-\cos \alpha=h \cos \zeta \cos \boldsymbol{H}$, vel $\boldsymbol{M}-\cos \left(\alpha^{\prime \prime}-\alpha\right)=h \cos \zeta \cos \left(\boldsymbol{H}-\alpha^{\prime \prime}\right)$ $\boldsymbol{M} \sin \alpha^{\prime \prime}-\sin \alpha=h \sin \zeta \sin \boldsymbol{H}$, vel $\left.\sin \left(\alpha^{\prime \prime}-\alpha\right)=h \sin \zeta \sin \left(\boldsymbol{H}-\alpha^{\prime \prime}\right)\right\}$ $\boldsymbol{M} \operatorname{tang} \delta^{\prime \prime}-\operatorname{tang} \delta=h \sin \zeta$, vel $\boldsymbol{M} \operatorname{tang} \delta^{\prime \prime}-\operatorname{tang} \delta=h \sin \zeta$ 
quarum ope $c$ sequentem obtinet valorem

$$
\begin{aligned}
c & =r\left\{(\rho h \cos \zeta \cos \boldsymbol{H}-\boldsymbol{g} \cos \boldsymbol{G})^{2}+(\rho \boldsymbol{h} \sin \zeta \sin \boldsymbol{H}-\boldsymbol{g} \sin \boldsymbol{G})^{2}+\rho^{2} h^{2} \sin ^{2} \zeta\right\} \\
& =\sqrt{ }\left\{\rho^{2} \boldsymbol{h}^{2}-2 \rho \boldsymbol{h} \boldsymbol{g} \cos \zeta \cos (\boldsymbol{G}-\boldsymbol{H})+\boldsymbol{g}^{2}\right\}
\end{aligned}
$$

quae formula ulteriorem haud abnuit simplificationem, si ponamus

quo reducitur ad hanc

$$
\begin{aligned}
& \cos \zeta \cos (\boldsymbol{G}-\boldsymbol{H})=\cos \phi \quad . \quad . \quad . \quad .(m), \\
& g \sin \phi=A \quad . \quad . \quad . \quad . \quad(n),
\end{aligned}
$$

ef ulterius progrediendo, si statuamus

$$
c=\checkmark\left\{(\rho h-g \cos \phi)^{2}+A^{2}\right\}
$$

$$
\rho h-g \cos \varphi=u, \text { quo fit } \rho=\frac{u+g \cos \varphi}{h} \cdot \cdot(0),
$$

ad hanc simplicissimam erimus perducti

$$
c=\boldsymbol{r}\left(\boldsymbol{u}^{2}+\boldsymbol{A}^{2}\right) \quad \cdot \quad \cdot \quad \cdot \quad \cdot \quad \cdot \quad \cdot(\boldsymbol{p}) .
$$

Expressiones ipsorum $r$ et $\boldsymbol{r}^{\prime \prime}$ simpliciores reddere etiam licet, statuendo

$$
\left.\begin{array}{c}
h \cos \delta=b \\
\frac{h \cos \delta^{\prime \prime}}{M}=b^{\prime \prime}
\end{array}\right\} .
$$

quo has formulas simplices pro $r$ et $\boldsymbol{r}^{\prime \prime}$ obtinemus

$$
\left.\begin{array}{rl}
r & =\sqrt{ }\left\{\left(\frac{u+s}{b}\right)^{2}+\boldsymbol{B}^{2}\right\} \\
\boldsymbol{r}^{\prime \prime} & =\sqrt{ }\left\{\left(\frac{u+s^{\prime \prime}}{b^{\prime \prime}}\right)^{2}+\boldsymbol{B}^{\prime \prime 2}\right\}
\end{array}\right\}
$$

Ex his formulis, quibus quantitates $r, \boldsymbol{r}^{\prime \prime}$ et $\boldsymbol{c}$ exprimuntur, $\boldsymbol{u}$ ita determinetur necesse est, ut exinde aequationi Lambertianae satisfiat

$$
\left(r+r^{\prime \prime}+c\right)^{\frac{3}{2}}-\left(r+r^{\prime \prime}-c\right)^{\frac{3}{2}}=\frac{t^{\prime \prime}-t}{m}
$$

denotante $m$ tempus dierum 9,6887401 et ideo $\log m=0,9862673$. Aequatio haec experimentis tali modo resolvitur, ut a valore ipsius $\boldsymbol{r}+\boldsymbol{r}^{\prime \prime}$ supposito egrediamur, cujus substitutione valorem correspondentem ipsius $c$ determinemus, quo $u, r$ et $r^{\prime \prime}$ ex aequationibus $(p)$ et $(s)$ eliciamus. Quo facto novus valor ipsius $r+\boldsymbol{r}^{\prime \prime}$ obtinetur, ex cujus congruentia cum valore supposito veritatem resultati concludere licet. Quum vero hoc plerumque non evenit, valor hic novus ipsius $r+\boldsymbol{r}^{\prime \prime}$ eodem modo adhibetur et calculus idemtidem repetitur, donec congruentia perfecta efficitur. Primum valorem adhibere possumus 
198 16. Bergius, de orbitis cometarum cx observationibus detcrmin. commentatio.

$r+\boldsymbol{r}^{\prime \prime}=2$, quum $\boldsymbol{r}+\boldsymbol{r}^{\prime \prime}<1$ esse non possit, quamdiu distantia apparens cometae a sole mojor quam $30^{\circ}$ est, quod fere semper evenit; et $r+r^{\prime \prime}>3$ perraro est, quoniam cometae nobis intra orbitam Martis plerumque sunt visibiles. Tali modo $u$ ea, quam obtinere possumus, perfectione computato, $\rho$ el $\rho^{\prime \prime}$ ex aequationibus supra tradilis determinantur.

\$. 6.

Quantitatibus $\rho$ et $\rho^{\prime \prime}$ jam cognitis facili negotio elementa orbitae eliciuntur. Ad hunc finem ponamus, exprimere

$\lambda, \lambda$ "longitudines cometae heliocentricas ad tempus primae et tertiae obcervationis ;

$\beta, \beta^{\prime \prime}$ latidudines heliocentricas;

$v, v^{\prime \prime}$ longitudines in orbita;

$\delta$ longitudinem nodi ascendenlis;

$i$ inclinationem orbitae, semper intra $0^{\prime \prime}$ el $90^{\prime \prime}$ contentam, si more usitato inter cometas, qui directe vel retro moventur, destinguamus:

$\pi$ longitudinem perihelii;

$\boldsymbol{T}$ tempus transitus per perihelium;

$q$ distantiam in perihelio.

$\mathrm{Si}$ projectiones locorum cometae in planum Ecliplicae consideremus, ope trigonometriae planae ad quantitates $\lambda, \beta, \lambda^{\prime \prime}, \beta^{\prime \prime}$ determinandas sequenles facillime colliguntur formulas

$$
\left.\begin{array}{rl}
\rho \cos (\alpha-\odot)-\boldsymbol{R} & =\boldsymbol{r} \cos \beta \cos (\lambda-\odot) \\
\rho \sin (\alpha-\odot) & =\boldsymbol{r} \cos \beta \sin (\lambda-\odot) \\
\rho \operatorname{tang} \delta & =\boldsymbol{r} \sin \beta \\
\rho^{\prime \prime} \cos \left(\alpha^{\prime \prime}-\odot{ }^{\prime \prime}\right)-\boldsymbol{R}^{\prime \prime} & =\boldsymbol{r}^{\prime \prime} \cos \beta^{\prime \prime} \cos \left(\lambda^{\prime \prime}-\odot \odot^{\prime \prime}\right) \\
\rho^{\prime \prime} \sin \left(\alpha^{\prime \prime}-\odot^{\prime \prime}\right) & =\boldsymbol{r}^{\prime \prime} \cos \beta^{\prime \prime} \sin \left(\lambda^{\prime \prime}-\odot^{\prime \prime}\right) \\
\rho^{\prime \prime} \operatorname{tang} \delta^{\prime \prime} & =\boldsymbol{r}^{\prime \prime} \sin \beta^{\prime \prime}
\end{array}\right\}
$$

Quoniam sex aequationes habemus, valores ipsorum $r$ et $\boldsymbol{r}^{\prime \prime}$ ex his etiam elicere possumus, quorum congruentia cum iis, ex quantitate $u$ deductis, veritatem calculi confirmat. Cometa directe vel retro movetur, prout $\lambda$ " majus minusve ipso $\lambda$ est.

Ad longitudinem nodi ascendentis et inclinationem orbitae determinandas ex principiis trigonometriae sphericae, si aequationem observemus identicam $\sin \left(\lambda^{\prime \prime}-\delta\right)=\sin \left((\lambda-\delta)+\left(\lambda^{\prime \prime}-\lambda\right)\right)$, sponte sequitur : 


$$
\left.\begin{array}{r} 
\pm \operatorname{tang} \beta=\operatorname{tang} i \sin (\lambda-\delta) \\
\pm \frac{\operatorname{tang} \beta^{\prime \prime}-\operatorname{tang} \beta \cos \left(\lambda^{\prime \prime}-\lambda\right)}{\sin \left(\lambda^{\prime \prime}-\lambda\right)}=\operatorname{tang} i \cos (\lambda-\delta)
\end{array}\right\}
$$

ubi signa adhibeantur superiora, si cometa directe moveatur, sin vero retro, inferiora.

Ad longitudines in orbita determinandas sine ullo negotio formulae deducuntur haecce

$$
\begin{aligned}
& \frac{\operatorname{tang}(\lambda-\delta)}{\cos i}=\operatorname{tang}(v-\delta) \\
& \frac{\operatorname{tang}\left(\lambda^{\prime \prime}-\delta\right)}{\cos i}=\operatorname{tang}\left(v^{\prime \prime}-\delta\right)
\end{aligned}
$$

Formulas motus parabolici supra traditas respicientibus formularum pro determinatione longitudinis perihelii et distantiae minimae deductio erit in aperto, si tantummodo observemus esse $\cos \frac{1}{2}\left(v^{\prime \prime}-\pi\right)=\cos \frac{1}{2}\left((v-\pi)+\left(v^{\prime \prime}-v\right)\right)$ :

$$
\begin{aligned}
& \frac{1}{\sqrt{r}}=\frac{1}{\sqrt{q}} \cos \frac{1}{2}(v-\pi) \\
& \left.\frac{\operatorname{cotg} \frac{1}{2}\left(v^{\prime \prime}-v\right)}{\sqrt{ } r}-\frac{1}{\sin \frac{1}{2}\left(v^{\prime \prime}-v\right) \sqrt{\prime \prime}}=\frac{1}{\sqrt{q}} \sin \frac{1}{2}(v-\pi)\right\}
\end{aligned}
$$

Ex tabulis Barkerianis ${ }^{1}$ ) (vel ex aliis tabulis motus cometarum) jam motus eliciuntur medios, anomaliis veris $v-\pi, v^{\prime \prime}-\pi$ vel $\pi-v, \pi-v^{\prime \prime}$ correspondentes; quos si litteris $\boldsymbol{N}, \boldsymbol{N}^{\prime \prime}$ designemus, duas expressiones temporis Iransitus cometae per perihelium obtinebimus sequentes:

$$
\boldsymbol{T}=t \mp \boldsymbol{N} n q^{\frac{3}{2}}=t^{\prime \prime} \mp \boldsymbol{N}^{\prime \prime} n q^{\frac{3}{2}}
$$

ubi signa valeant superiora, si motu directo $v>\pi, v^{\prime \prime}>\pi$ sit, vel si motus fiat retro, et sit $v<\pi, v^{\prime \prime}<\pi$; signa vero inferiora casus respiciunt oppositos. Constans est $n$ quantitas, cujus logarithmus $=0,0398723$ est. Duorum iqsius $\boldsymbol{T}$ concensus valorum veritatem confirmat calculi.

Ex elementis tali modo indagatis longitudo et latitudo geocentrica cometae pro tempore observationis mediae computantur, quorum valorum computatorum cum observatis consensus ultimam calculi trutinam suppeditet, ex qua judicare possumus, quanta cum accuratione elementa fuerint determinata.

Elementa orbitae hoc modo determinata non sunt nisi approximata. Superest igitur scrutari, quomodo ex hac prima elementorum determinatione ad cognitionem horum, quam observationes concedant, accuratissimam perveniri

1) Olbers, Abhandlung über die leichteste und bequemste Methode die Bahn eines Cometen aus einigen Beobachtungen zu berechnen. Weimar 1797. 
200 16. Bergius, de orbitis cometarum ex observationibus determin. commentatio.

licet. Priusquam autem hanc rem adgrediamur, superiorem juvat ad calculos numericos accommodare theoriam.

\$. 7

Computatio elementorum approximalorum cometae 28 Oct. 1842 Parisiis a Laugier inventi.

In observatorio Regio Berolinensi loci apparentes sunt observati sequentes:

1842 Nov. 5,326081 t. m. Berol. A. R. $268^{\prime \prime} 12^{\prime} 22^{\prime \prime}, 4$ Decl. +52 $14^{\prime} 53^{\prime \prime}, 1$

$$
\begin{aligned}
& 7,367911 \\
& 2713734,3 \\
& +46357,2 \\
& 8,370782 \\
& 273915,1 \\
& \alpha \quad .265^{\circ} 33^{\prime} 20^{\prime \prime}, 9 \quad \delta \quad .75^{\circ} 40^{\prime} 51^{\prime \prime}, 2 \\
& \begin{array}{llllllll}
\alpha^{\prime} & .273 & 13 & 27,4 & \delta^{\prime} & .69 & 30 & 28,9
\end{array} \\
& \alpha^{\prime \prime} .275 \quad 43 \quad 51,5 \quad \delta^{\prime \prime} .66 \quad 718,5
\end{aligned}
$$$$
+424327,2
$$

Ex tabulis porro colligitur astronomicis:

$$
\begin{aligned}
& \odot \quad .222^{\circ} 59^{\prime} 17^{\prime \prime}, 2 \quad \log \boldsymbol{R} \quad .9,9959650 \\
& \odot^{\prime} \ldots 225 \quad 2 \quad 22,9 \quad \log R^{\prime} \ldots 9,9957465 \\
& \odot^{\prime \prime} . .226 \quad 252,8 \quad \log R^{\prime \prime} . .9,9956406
\end{aligned}
$$

Quibus ex datis calculus hoc modo conficitur: ${ }^{1}$ )

$$
\begin{array}{rr}
\operatorname{tang} \delta^{\prime} . .0,42745 & \operatorname{tang} \delta . .0,59303 \\
\sin \left(\alpha-\odot^{\prime}\right) . .9,81268 & \sin \left(\alpha^{\prime}-\odot^{\prime}\right) . .9,87233 \\
\hline 0,24013 & 0,46536 \\
& \frac{0,39291}{0,07245} \\
& \frac{9,58745}{0,48500} \\
\operatorname{tang} \delta^{\prime \prime} .0,35391 & \operatorname{tang} \delta^{\prime} . .0,42745 \\
\sin \left(\alpha^{\prime}-\odot^{\prime}\right) . .9,87233 & \sin \left(\alpha^{\prime \prime}-\odot^{\prime}\right) \frac{.9,88860}{0,31605} \\
\hline 0,22624 & \frac{0,72860}{9,58745}
\end{array}
$$

1) Ut calcalus prodeat facilior, quinque tantummodo locos decimales adhibemus ct tabulas, quihus logarithmus summae et differentiae duorum numerorum ex datis horum logarithmis elicitur, in usum rocamus. 
16. Bergitus, de orbitis cometarum ex observationibus determin. commentatio. 201

$$
\begin{aligned}
& \log \left(\boldsymbol{t}^{\prime \prime}-t\right) . .0,00124 \quad \log \boldsymbol{R}^{\prime \prime} . .9,99564 \quad \log \boldsymbol{R}^{\prime \prime} . .9,99564 \\
& \log \left(t^{\prime}-t\right) \frac{.0,31002}{9,69122} \quad \cos \left(\odot^{\prime \prime}-\odot\right) \frac{. .9,99938}{9,99502} \quad \sin \left(\odot^{\prime \prime}-\odot\right) \frac{.88,72738}{8,72302}
\end{aligned}
$$

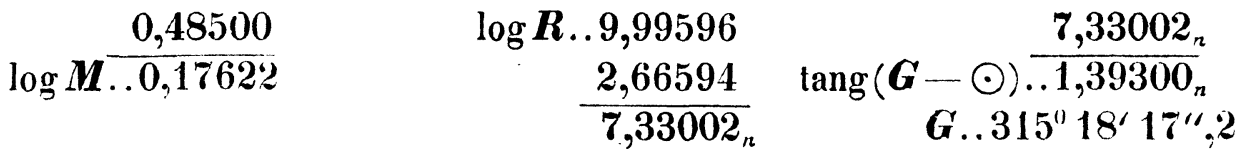

$$
\begin{aligned}
& 8,72302 \\
& \sin (\boldsymbol{G}-\odot) \frac{. .9,99964}{\log g . .8,72338} \\
& \cos \left(\alpha^{\prime \prime}-\alpha\right) . .9,99311 \\
& \frac{0,46342}{9,71280} \\
& \sin \left(\alpha^{\prime \prime}-\alpha\right) \ldots 9,24713 \\
& \operatorname{tang}\left(\boldsymbol{H}-\alpha^{\prime \prime}\right) . .9,53433 \\
& H-\alpha^{\prime \prime} . .18^{\prime \prime} 53^{\prime} 35^{\prime \prime} \\
& \text { H..292 } 72 \quad 2 \\
& \cos \zeta . .9,85639 \quad \cos \delta . .9,39326 \\
& \cos (\boldsymbol{G}-\boldsymbol{H}) . .9,96342 \\
& \cos \emptyset . .9,81981 \\
& \text { Ф..48" } 40^{\prime} 9^{\prime \prime} \\
& \begin{array}{r}
\cos (\alpha-\odot) . .9,86716 \\
\cos \psi . .9,26042 \\
\psi . .79^{\prime \prime} 30^{\prime} 19^{\prime \prime}
\end{array}
\end{aligned}
$$

$\log (\boldsymbol{g} \cdot \cos \varphi) . .8,45320 \log (\boldsymbol{b R} \cdot \cos \psi) . .8,53010 \log \left(\boldsymbol{b}^{\prime \prime} \boldsymbol{R}^{\prime \prime} \cos \psi^{\prime \prime}\right) . .8,72525$ num. $+0,03493 \quad$ num. $+0,03389 \quad$ num. $+0,05312$

$$
s_{. .}+0,00104 \quad s^{\prime \prime} \ldots-0,01819
$$

Jam sequitur, $\boldsymbol{u}$ ex aequationibus

$$
r=\sqrt{ }\left\{\left(\frac{u+s}{b}\right)^{2}+B^{\prime}\right\}, \quad r^{\prime \prime}=V\left\{\left(\frac{u+s^{\prime \prime}}{b^{\prime \prime}}\right)^{2}+B^{\prime \prime 2}\right\}, \quad k=V\left(u^{2}+A^{2}\right)
$$

experimentis ita determinare, ut aequationi $\left(\boldsymbol{r}+\boldsymbol{r}^{\prime \prime}+c\right)^{\frac{3}{2}}-\left(\boldsymbol{r}+\boldsymbol{r}^{\prime \prime}-c\right)^{\frac{3}{2}}=$ $\left(t^{\prime \prime}-t\right) 6 k$ satisfiat. Hoc ut facillime evadat ad $c$ computandum formulas 
202 16. Bergius, de orbitis cometarum ex obscrvationibus detcrmin. commentalio. auxiliares hasce adhibemus

$$
\sin \theta=\frac{6 k\left(t^{\prime \prime}-t\right)}{V 8\left(r+r^{\prime \prime}\right)^{\frac{3}{2}}}, \quad \mu=\frac{3 \sin \frac{1}{3} \theta}{\sin \theta} r\left(\cos \frac{2}{3} \theta\right), \quad c=\frac{2 k\left(t^{\prime \prime}-t\right)}{V\left(r+r^{\prime \prime}\right)} \mu .
$$

Si jam primum valorem approximativum (\$.5.) $\boldsymbol{r}+\boldsymbol{r}^{\prime \prime}=2$ statuamus, ex his formulis elicimus $\log \mu=0,00002$, ideoque

$$
\begin{aligned}
& \log 2 k . .8,53661 \\
& \log \left(t^{\prime \prime}-t\right) . .0,48354 \\
& \text { compl. } \log \left(\boldsymbol{r}+\boldsymbol{r}^{\prime \prime}\right)^{\frac{1}{2}} . .9,84949 \\
& \log \mu . .0,00002 \\
& \log c . .8,86966 \\
& \log A \text {. . 8,59898 } \\
& \log c . .8,86966 \\
& \log u . . \quad \frac{9,92639}{8,79605} \\
& u \text {.. }+0,06252 \\
& u+s \quad . .+0,06356 \\
& u+s^{\prime \prime} . .+0,04433
\end{aligned}
$$

$$
\begin{aligned}
& \log (u+s) . .8,80318
\end{aligned}
$$

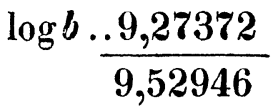

$$
\begin{aligned}
& \log \boldsymbol{B} . .9,98863 \\
& \log \left(u+s^{\prime \prime}\right) \ldots 8,64670 \\
& \log b^{\prime \prime} \frac{.9,31147}{9,33523} \\
& \log \boldsymbol{B}^{\prime \prime} . .9,98021
\end{aligned}
$$

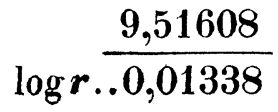

$$
\begin{aligned}
& \log \boldsymbol{r}^{\prime \prime} \frac{9,34416}{9,99107} \\
& \log \left(r+r^{\prime \prime}\right)_{1} .0,30340
\end{aligned}
$$

Jam cum hoc valore ipsius $r+\boldsymbol{r}^{\prime \prime}$ calculum eundem repetamus et eodem modo sequentes obtinemus valores

$$
\begin{array}{r}
\log \boldsymbol{A} . .8,59898 \\
\log c . .8,86847 \\
9,92590 \\
\log u . . \quad 8,79437 \\
u . .+0,06228 \\
u+s \quad . .+0,06332 \\
u+s^{\prime \prime} .+0,04409
\end{array}
$$


16. Bergius, de orbitis cometarum ex observationibus determin. commentatio. 203

$$
\begin{array}{rr}
\log (\boldsymbol{u}+s) . .8,80154 & \log \left(\boldsymbol{u}+\boldsymbol{s}^{\prime \prime}\right) . .8,64434 \\
\log \boldsymbol{b} . \mathbf{9 , 2 7 3 7 2} & \left.\log \boldsymbol{b}^{\prime \prime}\right) . .9,31147 \\
\mathbf{9 , 5 2 7 8 2} & \mathbf{9 , 3 3 2 8 7} \\
\log \boldsymbol{B} . .9,98863 & \log \boldsymbol{B}^{\prime \prime} . \mathbf{9 , 9 8 0 2 1} \\
\frac{9,97543}{\log \boldsymbol{. . 0 , 0 1 3 2 0}} & \log \boldsymbol{r}^{\prime \prime} . .9,989095 \\
\log \left(\boldsymbol{r}+\boldsymbol{r}^{\prime \prime}\right)_{2} . .0,30325
\end{array}
$$

Calculo denuo repetito invenilur:

$$
\begin{aligned}
& \log \boldsymbol{A} . .8,59898 \\
& \log c . .8,86855 \\
& 9,92594 \\
& \log u . . \quad 8,79449 \\
& u \text {.. }+0,06230 \\
& \begin{array}{r}
\log (\boldsymbol{u}+\boldsymbol{s}) . .8,80168 \\
\log \boldsymbol{b} \frac{.9,27372}{9,52796}
\end{array} \\
& \log \boldsymbol{B} . .9,98863 \\
& \log \left(u+s^{\prime \prime}\right) . .8,64454 \\
& \log b^{\prime \prime} \frac{.9,31147}{9,33307}
\end{aligned}
$$

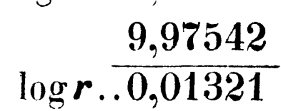

$$
\begin{aligned}
& \log \boldsymbol{B}^{\prime \prime} . .9,98021 \\
& \log \left(\boldsymbol{r}+\boldsymbol{r}^{\prime \prime}\right)_{3} . .0,30326
\end{aligned}
$$

Ex his ipsius $r+r^{\prime \prime}$ valoribus inventis interpolatione facillima ad verum appropinquare possumus. Ad hunc finem $r+r^{\prime \prime}=f$ ponamus, et hasce relationes supra adhibilas observamus

$$
\begin{gathered}
r+\boldsymbol{r}^{\prime \prime}=f_{0}, \quad c=\frac{2 k\left(t^{\prime \prime}-t\right)}{f_{\mathrm{o}}^{\frac{1}{2}}} \mu, \quad u=\sqrt{ }\left(c^{2}-\boldsymbol{A}^{2}\right) \\
r=\sqrt{ }\left\{\left(\frac{u+s}{b}\right)^{2}+\boldsymbol{B}^{2}\right\}, \quad \boldsymbol{r}^{\prime \prime}=\sqrt{ }\left\{\left(\frac{u+s^{\prime \prime}}{b^{\prime \prime}}\right)^{2}+\boldsymbol{B}^{\prime \prime 2}\right\}
\end{gathered}
$$

Si differentiemus, et $\mu$, quod tamen semper unitati proximum est, constans consideremus, relationes evadent sequentes:

$$
\begin{gathered}
\frac{d c}{c}=-\frac{1}{2} \frac{d f_{0}}{f_{0}}, \quad d u=\frac{c}{u} d c \\
r d r=\left(\frac{u+s}{b}\right) \frac{d u}{b}=r \cos C \frac{d u}{b} \\
r^{\prime \prime} d r^{\prime \prime}=\left(\frac{u+s^{\prime \prime}}{b^{\prime \prime}}\right) \frac{d u}{b^{\prime \prime}}=r^{\prime \prime} \cos C^{\prime \prime} \frac{d u}{b^{\prime \prime}}, \quad d r+d r^{\prime \prime}=d f_{\prime \prime},
\end{gathered}
$$


204 16. Bergius, de orbitis cometarum ex observationibus determin. commentatio.

ubi simplicitatis causa statuimus $\frac{u+s}{b}=\rho \sec \delta-\boldsymbol{R} \cos \psi=r \cos C$, et $\frac{u+s^{\prime \prime}}{b^{\prime \prime}}=\rho^{\prime \prime} \sec \delta^{\prime \prime}-\boldsymbol{R}^{\prime \prime} \cos \psi^{\prime \prime}=\boldsymbol{r}^{\prime \prime} \cos C^{\prime \prime} . \quad \boldsymbol{C}$ et $\boldsymbol{C}^{\prime \prime}$ igitur angulos exprimunt, quos lineae a loco cometae ad solem et tellurem ductae includunt. Porro adnolamus, esse $b=h \cos \delta, b^{\prime \prime}=\frac{h \cos \delta^{\prime \prime}}{M}$. Ex his sequitur

$$
\begin{aligned}
d r & =\quad \sec \delta \cos C \frac{d u}{h \varrho}=D \cos C \frac{d u}{h \varrho} \\
d r^{\prime \prime} & =\rho^{\prime \prime} \sec \delta^{\prime \prime} M \cos C^{\prime \prime} \frac{d u}{h \varrho^{\prime \prime}}=D^{\prime \prime} \cos C^{\prime \prime} \frac{d u}{h \varrho^{\prime \prime}}
\end{aligned}
$$

positis $\varrho \sec \delta=D$ et $M \rho^{\prime \prime} \sec \delta "=D "$. Quoniam nunc $d u=\frac{c}{u} d c$ est, evadel

$$
\begin{gathered}
d u=-\frac{1}{2} \frac{c^{2}}{u} \frac{d f_{0}}{f_{0}} \\
d f_{1}=-\frac{1}{2}\left(\frac{D \cos C+D^{\prime \prime} \cos C^{\prime \prime}}{r+r^{\prime \prime}}\right) \frac{c^{2}}{h \varrho u} d f_{0} .
\end{gathered}
$$

Haec expressio quum minus facile applicelur, observemus, quum loci cometae non procul a se invicem distant, esse $\boldsymbol{D}$ proxime $=D^{\prime \prime}$ et $\boldsymbol{C}$ proxime $=C^{\prime \prime}$. Quocirca medium inter illas quantitates adhibere possumus: quo evadet

$$
d f_{1}=-\frac{D^{\prime} \cos C}{r+r^{\prime \prime}} \cdot \frac{c^{2}}{h \rho u} d f_{11} \text {. }
$$

It $\rho^{h}$ eliminemus, relationem revocamus

$$
\boldsymbol{u}=\rho \boldsymbol{h}-\boldsymbol{g} \cos \varphi=\boldsymbol{c} \cos \chi_{\prime \prime},
$$

denotante $\chi_{\prime \prime}$ angulum, quem format linea, a primo loco cometae ad tertium ducta, cum illa, quae ducitur a tertio loco cometae ad punclum quoddam, respectu tertii loci telluris eodem modo positum, quo locus primus cometae respectu primi loci telluris. Ex his prodit

$$
d f_{1}=-\frac{D^{\prime} \cos C^{\prime}}{r+r^{\prime}} \cdot \frac{c}{c-g \cos \chi} d f_{0} .
$$

Quantitales in expressione ipsius $d f_{1}$ contentae in genere sunt positivae. Quae etiam in negativas abire possent, constantes sunt. Si $\boldsymbol{C}^{\prime}$ angulus sit obtusus, erit $\boldsymbol{r}^{\prime}<\boldsymbol{R}^{\prime}<1$. Si $\boldsymbol{c}-\boldsymbol{g} \cos \chi$ in quantitatem abiret negativam, $\boldsymbol{g}>\boldsymbol{c}$ esset, ex quo fit $\boldsymbol{r}^{\prime}>2$. Casus igitur duo, quibus signum negativum in positivum abiret, sunt illi rariores: si cometa solem et tellurem valde appropinquarel, vel procul a sole esset remotus. In genere signum igitur remanet negativum. Si igitur correctionem hoc modo exprimamus

$$
d f_{1}=-l d f_{0}
$$


16. Bergius, de orbitis cometarum ex observationibus determin. commentatio. 205 prima erit correctio negativa, secunda positiva, tertia negativa et sic porro

$$
\begin{aligned}
& d f_{1}=-l d f_{1} \\
& d f_{2}=-l d f_{1}=+l^{2} d f_{1} \\
& d f_{3}=-l d f_{2}=-l^{3} d f_{0} \\
& \text { etc. }
\end{aligned}
$$

Correctiones sunt igitur alternae positivae et negalivae, quod ad rapidiorem calculi approximationem erit utilissimum. Ut approximationem aestimemus, observamus esse

$$
l=-\frac{D^{\prime} \cos C^{\prime}}{r+r^{\prime}} \cdot \frac{c}{c-g \cos \chi}
$$

ubi $\boldsymbol{D}$, distantia a tellure, plerumque $<\boldsymbol{r}$ est et factor primus ideo fractus. Quod ad secundum adtinet factorem, observamus, $\chi$ esse 0 , si directiones motus cometae et telluris sibi invicem sint parallelae. $\chi$ vero est $180^{\circ}$, si directiones illae sibi invicem sint contrariae, quod plerumque evenit, quum cometae a tellure primum observantur. Vulgo igitur factor etiam posterior est fractus. Quocirca factores duo ipsius $l$ in genere fractiones sunt verae.

Si ad differentias abire velimus finitas habemus

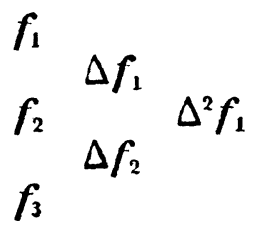

Quum jam est

$$
\begin{aligned}
f_{1} & =f-d f_{1} \\
f_{2} & =f-d f_{2} \\
\Delta f_{1} & =d f_{1}-d f_{2}=(1+l) d f_{1}
\end{aligned}
$$

eril

In genere fit $l$ post approximationem quandam constans. $\mathrm{Si}$ igitur plura sunt facta experimenta erit:

$$
d f_{1}=-l d f_{10}, \quad d f_{2}=-l d f_{1}=l_{2} d f_{0}
$$

quum vero

$$
\begin{array}{cl}
f=f_{1}+d f_{1}, & f=f_{2}+d f_{2}, \\
\Delta f_{1}=d f_{1}-d f_{2}=(1+l) d f_{1}, & \Delta f_{2}=(1+l) d f_{2}=-l(1+l) d f_{1},
\end{array}
$$
erit

$$
\frac{\Delta f_{2}}{\Delta f_{1}}=-l
$$

Hunc valorem ipsius $l$ inventum ad ulteriorem adhibere possumus approximationem. Quum

Crelle's Journal f. d. M. Bd. XXV. Heft 3. 
206 16. Bergius, de orbitis cometarum ex obscruationibus determin. commentalio.

$$
\Delta^{2} f_{1}=-(1+l)^{2} d f_{1}
$$

hoc modo $d f_{1}$ in functione ipsius $l$ expressum obtinemus siculi eliam $d f_{3}$. Prodit enim

$$
d f_{1}=-\frac{\left(\Delta f_{1}\right)^{2}}{\Delta^{2}}, \quad d f_{1}=-\frac{\left(\Delta / \dot{f}_{2}\right)^{2}}{\Delta^{2}},
$$

ex quibus illum polissimum eligamas coefficientem, qui numeratorem habet minimum.

Considerationem istam si ad exemplum nostrum adplicemus, valores supra inventos ipsius $\log \left(\boldsymbol{r}+\boldsymbol{r}^{\prime \prime}\right)=\boldsymbol{f}$ ad ulteriorem adhibeamus approximationem $\left.{ }^{1}\right)$

$$
\begin{gathered}
f_{1} . .0,30340 \\
f_{2} .00,30325-15+1+16 \\
f_{3} .00,30326 \\
\frac{\left(\Delta f_{2}\right)^{2}}{\Delta^{2}} f_{1}=0,00000, \quad d f_{3}=0, \text { et sic: } \\
f=0,30326
\end{gathered}
$$

Calculum si priorem cum hoc valore jam invento ipsius $f=\log \left(\boldsymbol{r}+\boldsymbol{r}^{\prime \prime}\right)$ iteremus evadent:

$$
\begin{array}{cc}
f . . & 0,30326 \\
\log c . . & 8,86854 \\
u . . & 0,0622991 \\
\log r . . & 0,013209 \\
\log r^{\prime \prime} . & 9,990966 \\
f_{1} . . & 0,303246
\end{array}
$$

calculoque denique repetito, hi resultant valores

$$
\begin{array}{cc}
\log c . . & 8,868529 \\
\boldsymbol{u} . .+ & 0,0622964 \\
\log \boldsymbol{r}_{. .} & 0,013208 \\
\log \boldsymbol{r}^{\prime \prime} . & \mathbf{9 , 9 9 0 9 6 0} \\
\boldsymbol{f}_{2} . & 0,303244
\end{array}
$$

Quod postremus ille valor ipsius $f$ a proximo duabus tantum unitalibus in ultimo loco differt, ex eo concludimus, valorem ipsius $r+\boldsymbol{r}^{\prime \prime}$ omni cum exactitudine, quam concedunt tabulae, esse inventum.

1) Eandem considerationem etiam ad logarithmos applicari posse, sponte intellegitur. 
$\Lambda d$ elementa orbitae aproximata determinanda hos igitur adhibeamus valores $\boldsymbol{u}=+0,0622964, \quad \log \boldsymbol{r}=0,013208, \quad \log \boldsymbol{r}^{\prime \prime}=9,990960$

Calculus sequens sic se habebit:

$$
\begin{aligned}
& u \ldots+0,0622964 \quad \log (u+g \cos \phi) . .8,987785 \\
& g \cos \varphi . .+0,0349302 \quad \log h . .9,88046 \\
& \log \rho . \overline{9,107325} \\
& \log \boldsymbol{M} . .0,17622 \\
& \log \rho^{\prime \prime} . \overline{\mathbf{9}, 283545} \\
& \cos (\alpha-\odot) \frac{.9,86716}{8,974485} \\
& \sin (\alpha-\odot) \frac{.9,83024}{8,937565} \\
& \log \rho . .9,107325 \\
& \cos \left(\alpha^{\prime \prime}-\odot^{\prime \prime}\right) \frac{.9,81091}{9,094455} \\
& \sin \left(\alpha^{\prime \prime}-\odot^{\prime \prime}\right) \frac{. .9,88223}{9,165775} \\
& \lambda "-\odot^{\prime \prime} . .170^{\circ} 23^{\prime 4} 47^{\prime \prime} .5 \quad \beta^{\prime \prime} . .26^{0} 18^{\prime} 3^{\prime \prime}, 1 \\
& \lambda^{\prime \prime . . ~} 36 \quad 26 \quad 40,3
\end{aligned}
$$

Ex eo, quod $\lambda^{\prime \prime}<\lambda$ est, concludimus, motum cometae esse relrogradum. Si his valoribus inventis $r$ et $r^{\prime \prime}$ computemus, obtinebimus: $\log r=0,013212$ et $\log r^{\prime \prime}=9,990965$, ex quo calculi veritatem concludimus. Porro fit:

tang $\beta . .9,745810 \quad$ tang $i \sin (\lambda-\delta) . .9,745810_{n}$ $0,543278_{n}$ $\cos (\lambda "-\lambda) \frac{.9,999930}{9,745740} \quad \begin{array}{rrr}\operatorname{tang} i \cos (\lambda-\delta) . .0,543278_{n} & \cos (\lambda-\delta) \ldots 9,994550^{n} \\ \operatorname{tang}(\lambda-\delta) . . \overline{9,202532} & \operatorname{tangi..0,538828}\end{array}$ $\operatorname{tang} \beta^{\prime \prime} . .9,693946$

$$
\begin{gathered}
\lambda-\delta . .189^{\circ} \quad 3^{\prime} 27^{\prime \prime . .5} \\
\delta . .208 \quad 24 \quad 49.1
\end{gathered}
$$$$
\text { i.. } 73^{\circ} 52^{\prime} 15^{\prime \prime}, 8
$$

$$
\frac{0,949143}{8,796597_{n}}
$$

$\sin (\lambda "-\lambda) \frac{.8,253319_{n}}{0,543278}$ 
208 16. Bergius, de orbitis cometarum ex observationibus determin. commentatio.

$$
\begin{aligned}
& \operatorname{lang}(\lambda-\delta) . .9,202532 \\
& \text { cosi.. 9,443732 } \\
& \text { tang }(v-\delta) . . \overline{9,758800} \\
& v-\delta . .209^{\circ} 50^{\prime} 58^{\prime \prime}, 0 \\
& \text { v.. } 58 \quad 1547,1 \\
& \text { cotang } \frac{1}{2}\left(v^{\prime \prime}-v\right) . .1,5930688_{n} \\
& \log \sqrt{ } \boldsymbol{r} . . \frac{0,006604}{1,5864648_{n}} \\
& -38,58912 \\
& +39,59028 \\
& +\longdiv { 1 , 0 0 1 1 6 } \\
& \begin{array}{c}
\operatorname{tang}\left(\lambda^{\prime \prime}-\delta\right) \ldots 9,149498 \\
\cos i . .9,443732 \\
\operatorname{tang}\left(v^{\prime \prime}-\delta\right) . .9,705766 \\
v^{\prime \prime}-\delta . .206^{\circ} 55^{\prime} 31^{\prime \prime}, 3 \\
v^{\prime \prime} . .55 \quad 2020,4
\end{array} \\
& \sin \frac{1}{2}\left(v^{\prime \prime}-v\right) . .8,4069314_{n} \\
& \log \sqrt{ } \boldsymbol{r}^{\prime \prime .99,995480} \\
& \overline{8,4024114_{n}} \\
& \text { compl. ..1,5975886 } \\
& -39,59028 \\
& \log \frac{1}{\sqrt{r}} \ldots 9,993396 \\
& \operatorname{tang} \frac{1}{2}(v-\pi) . .0,0,0071075 \\
& \frac{1}{2}(v-\pi) . .45^{\circ} 28^{\prime} \quad 7 ", 7 \\
& \pi \ldots 327 \quad 1931,7 \\
& \cos \frac{1}{2}(v-\pi) . .9,845902 \\
& \log \frac{1}{r_{q}} . \overline{0,147494} \\
& \log 9 . .9,705012
\end{aligned}
$$

$\log \varphi^{\frac{3}{2}} . .9,557518$

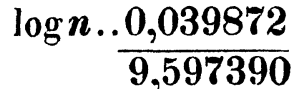

$\log N$..2,0107051

9,597390

40,55974

$$
\log N^{\prime \prime} \cdot \frac{1,9776034}{1,5749934}
$$

37,58317

T... $\frac{t 5,326081}{45,885821} \operatorname{dies}$

$\frac{8,370782}{45,953952}$ dies

Medium Nov. .. 45,919886

Elementa approximata orbitae comelae haec igitur sunt:

$$
\begin{array}{cccc}
T . .1842 & \text { December } & 15,919886 \\
\pi . .327^{\circ} & 19 & 31 ", 7 \\
8 . .208 & 24 & 49,1 \\
. . .73 & 52 & 15,8 \\
\log q . .9,705012 &
\end{array}
$$

Motus retrogradus. 
16. Bergius, de orbitis cometarum ex observationibus determin. commentatio. 209

Observator Petersen Altonae invenit : T..1842 Dec. 15,9643, $\pi . .327^{\circ} 37^{\prime} 21^{\prime \prime}$,

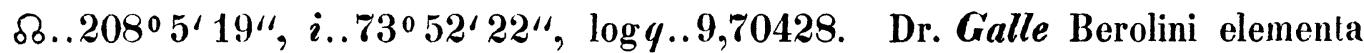
invenit haecce $T .1842$ Dec. $15,9726, \pi \ldots 327^{\circ} 30^{\prime} 4^{\prime \prime}, \delta . .208^{\circ} 1^{\prime} 36^{\prime \prime}$, i..73 $3^{\circ} 9^{\prime} 2^{\prime \prime}, \log q \ldots 9,70356$. Dom. Laugier Parisiis $T$. 1842 Dec. 15,8,

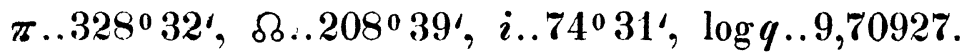

\section{\$. 8.}

Casus quidam dantur, in quibus methodus supra tradita applicari non potest, ii nempe, quum tres loci geocentrici cometae in eodem circulo maximo cum loco medio telluris apparentur, vel ad hunc valde approximantur; quoniam hoc casu numerator et denominator in expressione (e) ipsius $\boldsymbol{M}^{\prime}$ vel nihilo fiunt aequales, vel lam parvae evadunt, ut menda observationum maximam vim exercere possint. Casus illi excipiendi in natura ipsa problematis fontem habet suum. Si hoc eveniret, quod tamen rarius fit, ad expressiones (\$. 4.) hasce regrediendum est:

$$
\begin{aligned}
& {\left[\boldsymbol{r}^{\prime} \boldsymbol{r}^{\prime \prime}\right](\rho \cos \alpha-\boldsymbol{R} \cos \odot)-\left[\boldsymbol{r} \boldsymbol{r}^{\prime \prime}\right]\left(\rho^{\prime} \cos \alpha^{\prime}-\boldsymbol{R}^{\prime} \cos \odot^{\prime}\right)} \\
& +\left[\boldsymbol{r} \boldsymbol{r}^{\prime}\right]\left(\rho^{\prime \prime} \cos \alpha^{\prime \prime}-\boldsymbol{R}^{\prime \prime} \cos \odot{ }^{\prime \prime}\right)=0, \\
& {\left[\boldsymbol{r}^{\prime} \boldsymbol{r}^{\prime \prime}\right](\rho \sin \alpha-\boldsymbol{R} \sin \odot)-\left[\boldsymbol{r} \boldsymbol{r}^{\prime \prime}\right]\left(\rho^{\prime} \sin \alpha^{\prime}-\boldsymbol{R} \cdot \sin \odot{ }^{\prime}\right)} \\
& +\left[\boldsymbol{r} \boldsymbol{r}^{\prime}\right]\left(\rho^{\prime \prime} \sin \alpha^{\prime \prime}-\boldsymbol{R}^{\prime \prime} \sin \odot{ }^{\prime \prime}\right)=0, \\
& {\left[\boldsymbol{r}^{\prime} \boldsymbol{r}^{\prime \prime}\right] \rho^{\tan g} \delta-\left[\boldsymbol{r} \boldsymbol{r}^{\prime \prime}\right] \rho^{\prime} \operatorname{tang} \delta^{\prime}+\left[\boldsymbol{r} \boldsymbol{r}^{\prime}\right] \rho^{\prime \prime} \tan g \delta^{\prime \prime}=0 \text {. }}
\end{aligned}
$$

E duobus primis combinatione facillima deducere possumus:

$$
\begin{aligned}
{\left[\boldsymbol{r}^{\prime} \boldsymbol{r}^{\prime \prime}\right]\left(\rho \cos \left(\alpha-\odot^{\prime}\right)\right.} & \left.-\boldsymbol{R} \cos \left(\odot-\odot^{\prime}\right)\right)-\left[\boldsymbol{r} \boldsymbol{r}^{\prime \prime}\right]\left(\rho^{\prime} \cos \left(\alpha^{\prime}-\odot^{\prime}\right)-\boldsymbol{R}^{\prime}\right) \\
& +\left[\boldsymbol{r} \boldsymbol{r}^{\prime}\right]\left(\rho^{\prime \prime} \cos \left(\alpha^{\prime \prime}-\odot^{\prime}\right)-\boldsymbol{R}^{\prime \prime} \cos \left(\odot^{\prime \prime}-\odot^{\prime}\right)\right)=0, \\
{\left[\boldsymbol{r}^{\prime} \boldsymbol{r}^{\prime \prime}\right]\left(\rho \sin \left(\alpha^{\prime}-\alpha\right)\right.} & \left.+\boldsymbol{R} \sin \left(\odot-\alpha^{\prime}\right)\right)-\left[\boldsymbol{r} \boldsymbol{r}^{\prime \prime}\right] \boldsymbol{R}^{\prime} \sin \left(\odot^{\prime}-\alpha^{\prime}\right) \\
& -\left[\boldsymbol{r} \boldsymbol{r}^{\prime}\right]\left(\rho^{\prime \prime} \sin \left(\alpha^{\prime \prime}-\alpha^{\prime}\right)-\boldsymbol{R}^{\prime \prime} \sin \left(\odot^{\prime \prime}-\alpha^{\prime}\right)\right)=0 .
\end{aligned}
$$

Secunda harum aequationum suppeditat hanc ipsius $\varrho^{\prime \prime}$ expressionem:

$$
\begin{aligned}
& \rho^{\prime \prime}= \\
& \frac{\left[r^{\prime \prime} r^{\prime \prime}\right]}{\left[r r^{\prime}\right]} \cdot \frac{\sin \left(\alpha^{\prime}-\alpha\right)}{\sin \left(\alpha^{\prime \prime}-\alpha^{\prime}\right)} \cdot \rho+\frac{\left[r^{\prime \prime} r^{\prime \prime}\right] R \sin \left(\odot-\alpha^{\prime}\right)-\left[r r^{\prime \prime}\right] R \sin \left(\odot^{\prime}-\alpha^{\prime}\right)+\left[r^{\prime}\right] R^{\prime \prime} \sin \left(\odot^{\prime \prime}-\alpha^{\prime}\right)}{\left[r r^{\prime}\right] \sin \left(\alpha^{\prime \prime}-\alpha^{\prime}\right)} .
\end{aligned}
$$

Factores ipsorum $\left[\boldsymbol{r}^{\prime} \boldsymbol{r}^{\prime}\right],\left[\boldsymbol{r} \boldsymbol{r}^{\prime \prime}\right]$ et $\left[\boldsymbol{r} \boldsymbol{r}^{\prime}\right]$ in numeratore membri ultimi hujus expressionis, ut ordinatas solis, ad axem abscissarum relatas, cujus posilio per $\alpha^{\prime}$ est data, considerari possunt, quae per $\boldsymbol{Y}, \boldsymbol{Y}^{\prime}, \boldsymbol{Y}^{\prime \prime}$ designari est licitum. Respectu membrorum ordinis secundi habito, et posito: $k\left(t^{\prime \prime}-t^{\prime}\right)=\tau, k\left(t^{\prime \prime}-t\right)$ $=\tau^{\prime}, k\left(t^{\prime}-t\right)=\tau^{\prime \prime}$, hae relationes facillime deducuntur: 
210 16. Bergius, de orbitis cometarum cx observationibus detcrmin. cummentatio.

$$
\begin{aligned}
& \frac{\left[r^{\prime} r^{\prime \prime}\right]}{\left[r r^{\prime}\right]}=\frac{\left[R^{\prime} R^{\prime \prime}\right]}{\left[R R^{\prime}\right]}\left\{1-\frac{1}{6}\left(\tau^{2}-\tau^{\prime \prime 2}\right)\left(\frac{1}{r^{\prime 3}}-\frac{1}{R^{\prime 3}}\right)\right\}, \\
& \frac{\left[r r^{\prime \prime}\right]}{\left[r^{\prime \prime}\right]}=\frac{\left[R R^{\prime \prime}\right]}{\left[R R^{\prime}\right]}\left\{1-\frac{1}{6}\left(\tau^{\prime 2}-\tau^{\prime \prime 2}\right)\left(\frac{1}{r^{\prime 3}}-\frac{1}{R^{\prime 3}}\right)\right\} .
\end{aligned}
$$

His valoribus in ultimo membro substilutis, in numeratore erit expressio, quae sequenti modo scribi potest:

$$
\left[\boldsymbol{R}^{\prime} \boldsymbol{R}^{\prime \prime}\right] \boldsymbol{Y}-\left[\boldsymbol{R} \boldsymbol{R}^{\prime \prime}\right] \boldsymbol{Y}^{\prime}+\left[\boldsymbol{R} \boldsymbol{R}^{\prime}\right] \boldsymbol{Y}^{\prime \prime}
$$

quae ex (12) nihilo est aequalis. Restant tantummodo termini cum $\frac{1}{r^{\prime 3}}-\frac{1}{R^{\prime 3}}$ multiplicati. Si in his pro $\frac{\left[\boldsymbol{R}^{\prime} \boldsymbol{R}^{\prime \prime}\right]}{\left[\boldsymbol{R} \boldsymbol{R}^{\prime}\right]}, \frac{\left[\boldsymbol{R} \boldsymbol{R}^{\prime \prime}\right]}{\left[\boldsymbol{R} \boldsymbol{R}^{\prime}\right]}$ valores substituantur approximati $\frac{\tau}{\tau^{\prime \prime}}, \frac{\tau^{\prime}}{\tau^{\prime \prime}}$. terminis ordinum superiorum neglectis, illa prodibit ipsius $\varrho^{\prime \prime}$ expresio:

$$
\rho^{\prime \prime}=\frac{t^{\prime \prime}-t^{\prime}}{t^{\prime}-t} \cdot \frac{\sin \left(\alpha^{\prime}-\alpha\right)}{\sin \left(\alpha^{\prime \prime}-\alpha^{\prime}\right)} \rho-\frac{1}{2} \tau \tau^{\prime} \frac{\sin \left(\alpha^{\prime}-\odot^{\prime}\right)}{\sin \left(\alpha^{\prime \prime}-\alpha^{\prime}\right)}\left(\frac{1}{r^{\prime 3}}-\frac{1}{R^{\prime 3}}\right) R^{\prime} .
$$

Hoc in casu a valore approximato ipsius $\boldsymbol{M}$ progrediendum est, quo calculus ad finem experimentorum perducitur, et inde valores approximati ipsorum $r$ et $\boldsymbol{r}^{\prime \prime}$ determinantur. Jam vero valor ipsius $\boldsymbol{r}^{\prime \prime}$ interpolatione deducitur. His substitutis valoribus ad accuratiorem ipsius $\boldsymbol{M}$ valorem pervenitur, quocum calculus ad finem plerumque perduci potest, quamvis in hoc casu exactitudo determinationis elementorum orbitae semper magis fil circumscripta, quam in eo, quo methodus adhiberi potest directa.

\section{\$. 9.}

Superest jam examinare, quomodo ex elementis tali modo proxime determinatis elementa orbitae omni, quam concedant observationes, cura correcta deducuntur.

Ad hunc finem tres inter se distantes eligunlur observationes. Ope elementorum jam proxime determinatorum veras computantur anomalias correspondentes $v, v^{\prime}, v^{\prime \prime}$ radiique vectores $r, r^{\prime}, r^{\prime \prime} ; v^{\prime}-v$ et $v^{\prime \prime}-\boldsymbol{v}$ angulos igitur, inter radios vectores $\boldsymbol{r}$ et $\boldsymbol{r}^{\prime}, \boldsymbol{r}$ et $\boldsymbol{r}^{\prime \prime}$ inclusos exprimunt. Si elementa jamjam inventa vera sint, valores computati ipsorum $\boldsymbol{v}^{\prime}-\boldsymbol{v}$ et $\boldsymbol{v}^{\prime \prime}-\boldsymbol{v}$ cum iis, directe ex observationibus deductis, congruant necesse est; sicut vice versa differentia horum binorum valorum ex elementis minus recte suppositis existere debet.

Ex observationibus hoc modo $\boldsymbol{v}^{\prime}-\boldsymbol{v}$ et $\boldsymbol{v}^{\prime \prime}-\boldsymbol{v}$ deducuntur. Unaquaque observatione longitudo et latitudo geocentrica, $\alpha$ et $\delta$, obtinentur. Ut ex illis longitudinem et latitudinem heliocentricam $\lambda$ et $\beta$ derivemus. ponamus, $\boldsymbol{S}$, 
$\boldsymbol{T}$ et $\boldsymbol{C}$ locos respectivos exprimere solis, telluris et cometae. Si igitur designemus angulum $\boldsymbol{C} \boldsymbol{T} \boldsymbol{S}$ per $\gamma$, erit

$$
\cos \gamma=\cos (\alpha-\odot) \cos \delta
$$

quoniam $\boldsymbol{S} \boldsymbol{T} \boldsymbol{C}^{\prime}=\alpha-\odot$. In triangulo $\boldsymbol{C T S}$ duo supponimus latera $\boldsymbol{R}$ el $\boldsymbol{r}$ cognita, sicuti angulus $\gamma$ lateri $\boldsymbol{r}$ oppositus; quocirca angulus $\mathbf{S C T}=\eta$ delerminetur ex relatione

$$
\sin \eta=\frac{\boldsymbol{R}}{\boldsymbol{r}} \sin \gamma
$$

et deinceps est etiam angulus $\boldsymbol{C S T}=u$ cognitus. In pyramide triangulari $\left.C C_{1} S^{T}{ }^{1}\right)$ habemus :

$$
\sin \beta=\frac{\sin \delta \sin \psi}{\sin \gamma}, \quad \cos C_{1} \boldsymbol{S} \boldsymbol{T}=\frac{\cos \psi}{\cos \beta} .
$$

Designet jam $\boldsymbol{L}$ longitudo telluris heliocentrica, erit $\gamma=\boldsymbol{L}+\boldsymbol{C}_{1} \boldsymbol{S} \boldsymbol{T}$. Hoc igitur modo longitudines et latitudines cometae heliocentricae pro tribus observationum temporibus determinantur. Sint $\boldsymbol{C} \boldsymbol{C}^{\prime} \boldsymbol{C}^{\prime \prime}$ loci cometae his ipsis temporibus, $\boldsymbol{C}_{1} \boldsymbol{C}_{\mathbf{1}} \cdot \boldsymbol{C}_{\mathrm{1}}$ " projectiones horum punctorum in planum eclipticae, erint in triangulo spherico, quem formant puncta $\boldsymbol{C}$ et $\boldsymbol{C}^{\prime}$ cum polo eclipticae, duo latera $90^{\prime \prime}-\beta$ et $90^{\prime \prime}-\beta^{\prime}$ cognita, sicuti angulus polaris $\lambda^{\prime}-\lambda$; et relationes prodibunt haecce:

$$
\left.\begin{array}{l}
\cos \left(v^{\prime}-v\right)=\cos \left(\lambda^{\prime}-\lambda\right) \cos \beta \cos \beta^{\prime}+\sin \beta \sin \beta^{\prime} \\
\cos \left(v^{\prime \prime}-v\right)=\cos \left(\lambda^{\prime \prime}-\lambda\right) \cos \beta \cos \beta^{\prime \prime}+\sin \beta \sin \beta^{\prime \prime}
\end{array}\right\} \cdot\left(a^{\prime}\right) .
$$

Differentiae inter hos valores ipsorum $v^{\prime}-v$ et $v^{\prime \prime}-\boldsymbol{v}$ et illos, qui ex elementis fuerunt deducti, ex elementis minus accuratis existunt. Sint igitur $m^{\prime}$ et $m^{\prime \prime}$ valores ipsorum $v^{\prime}-v$ et $v^{\prime \prime}-v$ ex elementis deducti, ut etiam $\mu$ et $\mu^{\prime \prime}$ valores harum quantitatum superiori modo determinati. Sint porro $\delta m^{\prime}$, $\delta m^{\prime \prime}, \delta \mu^{\prime}, \delta \mu^{\prime \prime}$ variationes, quae in $m^{\prime}, m^{\prime \prime}, \mu^{\prime}, \mu^{\prime \prime}$ ex correctionibus elementorum ipsorum nascuntur, erint

$$
\left.\begin{array}{l}
m^{\prime}+\delta m^{\prime}=\mu^{\prime}+\delta \mu^{\prime} \\
m^{\prime \prime}+\delta m^{\prime \prime}=\mu^{\prime \prime}+\delta \mu^{\prime \prime}
\end{array}\right\}
$$

His duabus aequationibus correctiones, quae tempori transitus cometae per perihelium et distantiae minimae sunt applicandae, ut observationibus satisfiant, obtinentur. His autem elementis accurate determinatis, eadem cum exactitudine ex iis cetera deduci elementa est licitum.

Ut aequationes (b) evolvamus formulas motus parabolici, supra (\$. 3.) inventas, revocamus

1) $C_{1}$ est projectio puncti $\boldsymbol{C}$ in planum eclipticae. 
212 16. Bcrgius, de orbitis cometarum ex observationibus determin. commentatio.

$$
\left.\begin{array}{l}
r=\frac{q}{\cos ^{2} \frac{1}{2} v} \\
t=q^{\frac{3}{2}} V^{2}\left(\operatorname{tang} \frac{1}{2} v+\frac{1}{3} \operatorname{tang}^{3} \frac{1}{2} v\right)
\end{array}\right\}
$$

el supponimus, tempus transitus per perihelium minimamque distantiam minimas per $\delta$ designatas variationes subire; facla variatione elicitur

$$
\left.\begin{array}{l}
\frac{\delta r}{r}=\frac{\delta q}{q}+\operatorname{tang} \frac{1}{2} v \delta v \\
\delta v=\frac{V(2 q)}{r^{2}}\left(\frac{\delta t}{t}-\frac{3}{2} \frac{\delta q}{q}\right) \cdot t
\end{array}\right\}
$$

quibus varialiones radii vectoris et anomaliae verae, variationibus ipsorum $q$ et $\boldsymbol{t}$ correspondentes, determinantur. Ut variationes longitudinis et latitudinis heliocentricae, $\delta \lambda$ et $\delta \beta$ consequamur, expressiones ipsorum $\beta$ et $\boldsymbol{C}_{1} \boldsymbol{S T}$ supra inventas variemus, et retineamus, $\delta$ et $\gamma$ non variari, et esse $\delta \lambda=\delta . \boldsymbol{C}_{1} \boldsymbol{S T}$; quo fit

$$
\delta \beta=\operatorname{tang} \beta \operatorname{cotang} u . \delta u \text {. }
$$

Jam vero est

ideoque

$$
\begin{aligned}
& \delta \eta=-\operatorname{tang} \eta \frac{\delta r}{r} \\
& \delta \varkappa=-\delta \eta=\operatorname{tang} \eta \frac{\delta r}{r},
\end{aligned}
$$

$$
\delta \beta=\operatorname{tang} \beta \operatorname{tang} \eta \operatorname{cotang} \varkappa \frac{\delta r}{r} \quad . \cdots \quad\left(e^{\gamma}\right) .
$$

Pro longitudine etiam facillime deducimus:

$$
\begin{aligned}
\delta \lambda & =\cos C_{1} \boldsymbol{S T}(\operatorname{tang} u \delta u-\operatorname{tang} \beta \delta \beta) \\
& \left.=\operatorname{cotang} C_{1} \boldsymbol{S T}\left(\operatorname{tang} u \operatorname{tang} \eta \frac{\delta r}{r}-\operatorname{tang} \beta \delta \beta\right)\right\} \cdot .\left(f^{\prime}\right) .
\end{aligned}
$$

Ex his formulis $\left(e^{\prime}\right)$ et $\left(f^{\prime}\right)$ variationes longitudinum et latitudinum heliocentricarum deducuntur pro tribus observationibus, quas ad elementa corrigenda elegimus. Quas variationes, si valores ex $\left(d^{\prime}\right)$ substituantur, in functionibus ipsarum $\delta q$ et $\delta t$ exprimentur.

Quum vero supra $\left(a^{\prime}\right)$ invenimus

$$
\cos \mu^{\prime}=\cos \left(\lambda^{\prime}-\lambda\right) \cos \beta \cos \beta^{\prime}+\sin \beta \sin \beta^{\prime},
$$

ad faciliorem ipsius $\mu^{\prime}$ computationem angulum $\sigma$ introducamus axiliarem, ut fit

$$
\sin ^{2} \frac{1}{2} \sigma=\cos ^{2} \frac{1}{2}\left(\lambda^{\prime}-\lambda\right) \cos \beta \cos \beta^{\prime},
$$

quo fit:

$$
\delta \sigma=-\operatorname{tang} \frac{1}{2} \sigma\left\{\operatorname{tang} \frac{1}{2}\left(\lambda^{\prime}-\lambda\right)\left(\delta \lambda^{\prime}-\delta \lambda\right)+\operatorname{tang} \beta \delta \beta+\operatorname{tang} \beta^{\prime} \delta \beta^{\prime}\right\},
$$


16. Bergius, de orbitis cometarum ex observationibus determin. commentatio. 213

et exinde

quo prodibit

$$
\sin ^{2} \frac{1}{2} \mu^{\prime}=\cos \frac{1}{2}\left(\beta+\beta^{\prime}+\sigma\right) \cos \frac{1}{2}\left(\beta+\beta^{\prime}-\sigma\right)
$$

$\delta \mu^{\prime}=-\frac{1}{2} \operatorname{tang} \frac{1}{2} \mu^{\prime}\left\{\operatorname{tang} \frac{1}{2}\left(\beta+\beta^{\prime}+\sigma\right)\left(\delta \beta+\delta \beta^{\prime}+\delta \sigma\right)\right.$

$$
\left.+\operatorname{tang} \frac{1}{2}\left(\beta+\beta^{\prime}-\sigma\right)\left(\delta \beta+\delta \beta^{\prime}-\delta \sigma\right)\right\} \quad\left(g^{\prime}\right) \text {. }
$$

Si in $\left(g^{\prime}\right)$ valores quantitatum $\delta \beta, \delta \beta^{\prime}, \delta \sigma$ supra inventos substituamus, quanlitatem $\delta \mu^{\prime}$ per $\delta q$ et $\delta t$ expressam obtinebimus.

Ut eliam $\delta m$ per easdem exprimatur quantitates, recordari solummodo est opus, esse $\delta m=\delta v^{\prime}-\delta v$, et quantitates $\delta v^{\prime}$ et $\delta v$ ex $\left(d^{\prime}\right)$ determinari.

Duas igitur obtinebimus hujus formae aequationes

$$
\left.\begin{array}{l}
\delta \mu^{\prime}=f . \delta q+g . \delta t \\
\delta m^{\prime}=h . \delta q+k . \delta t
\end{array}\right\}
$$

quum vero est $\delta \mu^{\prime}-\delta m^{\prime}=m^{\prime}-\mu^{\prime}$, erit

$$
(f-h) \delta q+(g-k) \delta t=m^{\prime}-\mu^{\prime}
$$

Eodem modo primae et tertiae observationis combinatione consequimur

$$
\left(f^{\prime}-k^{\prime}\right) \delta q+\left(g^{\prime}-k^{\prime}\right) \delta t=m^{\prime \prime}-\mu_{\prime}^{\prime \prime} . \quad . \quad\left(k_{1}^{\prime}\right),
$$

ex quibus duabus aequationibus $\left(k^{\prime}\right)$ et $\left(k_{1}{ }^{\prime}\right)$ quantitates incognitae $\delta q$ et $\delta t$ eliminatione deducuntur.

Jam distantia in perihelio et tempus transitus accurate essent determinata, si calculus fuisset rigorosus. In aequationibus $(k)$ vero formandis quantitates ordinis secundi fuerunt neglectae, quocirca calculum cum elementis ita correclis idemtidem renovari necesse erit, quousque valores quantitatum $q$ et $t$ oblineantur, qui tam prope, quam fieri potest, observationibus satisfaciunt; ad quem finem tres plerumque requiruntur calculi repetitiones.

Quum tali modo distantia in perihelio et tempus transilus summa cum diligentia fuerunt determinata, ex his cetera eodem gradu exactitudinis facillime computantur elementa. Valoribus scilicet ipsorum $\delta q$ et $\delta t$, ultima calculi operatione consecutis, in aequationibus $\left(e^{\prime}\right)$ et $(f)$ substitutis, valores compulantur correcti longitudinum latitudinumque heliocentricarum $\lambda$ et $\beta$ pro tribus temporibus observationum. Harum ope inclinatio orbitae et longitudo nodi ascendentis et denique cetera elementa ex formulis in $\$$. 6 . tradilis eliciuntur.

In casibus evenire potest specialibus, ut elementa hoc modo correcta non nisi imperfecte observationibus satisfaciant, ex quo concludere licet, cometam non in parabola, ut supposuimus, moveri, sed in ellipsi vel hyperbola. Orbitae hyperbolicae in Astronomia minoris sunt momenti. 
214 16. Bergius, de orbitis cometarum ex obscrvationibus determin. commentatio.

\$. 10.

Suppositio orbilae parabolicae cometarum rigorose non est vera et, infinitis casibus, qui orbitam efficiunt elipticam vel hyperbolicam, cum iis, qui parabolicam producunt, comparatis, parum verosimilis. Quum ceterum comela, qui in orbita moveatur parabolica vel elliptica, semel tantummodo nobis sit visibilis, probabiliter adoptare possumus, cometas, qui in orbitis talibus moventur, si alioquin existant, jam pridem perihelium suum transiisse, nos vero nunc temporis cometas tantummodo observare, qui longiore vel breviore lemporis intervallo orbitas suas circa solem describant, qui ceteroquin Astronomiae maximi sunt momenti.

Si copia bonarum observationum ante et post transitum comelae per perihelium reperiatur, sequenti modo aliqua cum probabilitate tempus revolutionis determinari potest. Ponamus, parabolam, quae proxime omnibus satisfaciant observalionibus, fuisse determinatam, et $v, v^{\prime}, v^{\prime \prime}, v^{\prime \prime}$ etc. anomalias esse veras, sicuti $\boldsymbol{r}, \boldsymbol{r}^{\prime}, \boldsymbol{r}^{\prime \prime}, \boldsymbol{r}^{\prime \prime \prime}$ etc. radios vectores temporibus observationum correspondentes; sint porro $v^{\prime}-v=m, v^{\prime \prime}-v=m^{\prime}, v^{\prime \prime}-v=m^{\prime \prime}$ etc.: ex methodo superiori $m, m^{\prime}, m^{\prime \prime}$ etc. $\mu, \mu^{\prime}, \mu^{\prime \prime}$ etc. computantur. Posito:

$$
m-\mu^{\prime}=\boldsymbol{M}, \quad m^{\prime}-\mu^{\prime}=\boldsymbol{M}^{\prime}, \quad m^{\prime \prime}-\mu^{\prime \prime}=\boldsymbol{M}^{\prime \prime}, \quad m^{\prime \prime \prime}-\mu^{\prime \prime \prime}=\boldsymbol{M}^{\prime \prime \prime} \text { elc. }
$$

Si jam distantia in perihelio quantilate minima variatur, et hoc sit casu:

$$
m-\mu^{\prime}=\mathbf{N}, \quad m^{\prime}-\mu^{\prime}=\mathbf{N}^{\prime}, \quad m^{\prime \prime}-\mu^{\prime \prime}=\mathbf{N}^{\prime \prime}, \quad m^{\prime \prime}-\mu^{\prime \prime \prime}=\mathbf{N}^{\prime \prime} \text { elc. }
$$

Alia in hopothesi tempus transitus quantitate variatur perparva, distantia in perihelio eadem retenta, quae in primo erat casu; et sit jam:

$$
m-\mu^{\prime}=\boldsymbol{P}, \quad m^{\prime}-\mu^{\prime}=\boldsymbol{P}, \quad m^{\prime \prime}-\mu^{\prime \prime}=\boldsymbol{P}^{\prime \prime}, \quad m^{\prime \prime}-\mu^{\prime \prime}=\boldsymbol{P} "{ }^{\prime \prime} \text { elc. }
$$

Denique retineantur distantia in perihelio et tempus transitus, qualia in primo erant casu, computentur autem anomalia vera $v$ et radius vector $r$, orbita supposita elliptica, in qua excentricitas $e$ proxime est unitati aequalis, ut differentia $1-e$ perparva assumi potest. Ut valor ipsius $v$ obtineatur, nihil aliud est opus, quam ut ad valorem, primo casu in parabola computatum, angulus addatur parvus, cujus sinus est

$$
\frac{1}{10}(1-e) \cdot \operatorname{tang} \frac{1}{2} v\left\{4-3 \cos ^{2} \frac{1}{2} v-6 \cos ^{4} \frac{1}{2} v\right\} \text {. }
$$

Hoc valore ipsius $v$ introducto in formula

$$
r=\frac{\eta}{\cos ^{2} \frac{1}{2} v}\left\{1-\frac{1-e}{2} \operatorname{tang}^{2} \frac{1}{2} v\right\}
$$

valor ebtinetur correspondens ipsius $r$.

Eodem determinantur modo $v^{\prime}, r^{\prime}, v^{\prime \prime}, r^{\prime \prime}, v^{\prime \prime}, r^{\prime \prime}$ etc. ex quibus $m$, $m^{\prime}, m^{\prime \prime}, m^{\prime \prime \prime}$ etc. $\mu, \mu^{\prime}, \mu^{\prime \prime}, \mu^{\prime \prime \prime}$ etc. Sit hoc casu: 
16. Bergius, de orbitis cometarum ex observationibus determin. commentatio. 215

$$
m-\mu=Q, \quad n^{\prime}-\mu^{\prime}=Q^{\prime}, \quad m^{\prime \prime}-\mu^{\prime \prime}=Q^{\prime \prime}, \quad m^{\prime \prime}-\mu^{\prime \prime \prime}=Q^{\prime \prime} \text { etc. }
$$

Sit $u$ numerus, cum quo varialio supposita distantiae in perihelio multiplicari debet, ut vera prodeat, $t$ numerus, quocum variatio temporis transitus multiplicelur, $s$ vero numerus, quocum valor suppositus ipsius $1-e$ multiplicari debet, ut prodeat verus; sequentes formentur aquationes:

$$
\begin{gathered}
(\boldsymbol{M}-\boldsymbol{N}) u+(\boldsymbol{M}-\boldsymbol{P}) t+(\boldsymbol{M}-\boldsymbol{Q}) s=\boldsymbol{M} \\
\left(\boldsymbol{M}^{\prime}-\boldsymbol{N}^{\prime}\right) \boldsymbol{u}+\left(\boldsymbol{M}^{\prime}-\boldsymbol{P}^{\prime}\right) \boldsymbol{t}+\left(\boldsymbol{M}^{\prime}-\boldsymbol{Q}^{\prime}\right) \boldsymbol{s}=\boldsymbol{M}^{\prime} ; \\
\left(\boldsymbol{M}^{\prime \prime}-\boldsymbol{N}^{\prime \prime}\right) \boldsymbol{u}+\left(\boldsymbol{M}^{\prime \prime}-\boldsymbol{P} "\right) \boldsymbol{t}+\left(\boldsymbol{M}^{\prime \prime}-\boldsymbol{Q}^{\prime \prime}\right) s=\boldsymbol{M}^{\prime \prime} \\
\left(\boldsymbol{M}^{\prime \prime}-\boldsymbol{N}^{\prime \prime}\right) \boldsymbol{u}+\left(\boldsymbol{M}^{\prime \prime}-\boldsymbol{P}^{\prime \prime}\right) \boldsymbol{t}+\left(\boldsymbol{M}^{\prime \prime}-\boldsymbol{Q}^{\prime \prime}\right) s=\boldsymbol{M}^{\prime \prime} ; \\
\text { etc. }
\end{gathered}
$$

ex quibus valores ipsorum $u, t, s$ quam proxime his omnibus aequalionibus satisfacientes methodo solita determinantur, et tali modo vera distantia in perihelio, verum tempus transilus per perihelium et verus ipsius $1-e$ valor consequentur. Semiaxis major erit jam $\frac{q}{1-c}$, et tempus revolutionis $=\left(\frac{q}{1-c}\right)^{\frac{3}{2}}$, distanlia media solis unitati aequali posita.

Quam exacte autem quis suas instituat observationes, tanta tamen in tempore revolutionis definiendo restat incertitudo, ut ea solum via certum tempus revolutionis definietur, si cometa idem ad perihelium suum revertens observetur.

Corrigendum.

Pag. 201 lin. 28 pro 8,45320 lege 8,54320 